\title{
ON A MULTISCALE A POSTERIORI ERROR ESTIMATOR FOR THE STOKES AND BRINKMAN EQUATIONS
}

\author{
RODOLFO ARAYA, RAMIRO REBOLLEDO, AND FRÉDÉRIC VALENTIN
}

\begin{abstract}
This work proposes and analyzes a residual a posteriori error estimator for the Multiscale Hybrid-Mixed (MHM) method for the Stokes and Brinkman equations. The error estimator relies on the multi-level structure of the MHM method and considers two levels of approximation of the method. As a result, the error estimator accounts for a first-level global estimator defined on the skeleton of the partition and second-level contributions from element-wise approximations. The analysis establishes local efficiency and reliability of the complete multiscale estimator. Also, it yields a new face-adaptive strategy on the mesh's skeleton which avoids changing the topology of the global mesh. Specially designed to work on multiscale problems, the present estimator can leverage parallel computers since local error estimators are independent of each other. Academic and realistic multiscale numerical tests assess the performance of the estimator and validate the adaptive algorithms.
\end{abstract}

\section{INTRODUCTION}

The Stokes and Brinkman equations are widely used in applications involving multiple scales as found in geosciences (e.g., reservoirs computations) and biomedical engineering (e.g., blood flow simulations) problems, to cite a few. In such a scenario, exact pressure and velocity may show highly changing behaviors either due to highly heterogeneous or singularly perturbed physical coefficients, or when the media presents cavities and fractures as those structures can dramatically change the flow field. Standard finite element methods must rely on very fine meshes to capture such multiscale features of the velocity and pressure solutions, which yields to costly linear systems. This fact makes realistic three-dimensional simulations prohibitive even on modern parallel computers.

Multiscale finite element methods became an attractive alternative to simulate complex phenomena on coarse meshes. Behind the multiscale methods is the fundamental idea that base functions can upscale unresolved multiscale structures. Such basis satisfies local problems which resemble the original one, and then, they incorporate real coefficients and element's geometry in their design. This strategy was first proposed and analyzed in [8] for a one-dimensional Poisson problem and further extended to higher dimensions in [28]. Since, several variants of it have been proposed for fluid flow problems as the Heterogeneous Multiscale method (HMM) [38], the Variational Multiscale method (VMS) [29], the Generalized Multiscale finite element method [18], and the Localized Orthogonal Decomposition method (LOD) [32], to mention a few. For some of those methods a posteriori error analysis is provided, see for instance $[9,30,1,33,14,27,36,34]$ and the references therein.

The Multiscale Hybrid-Mixed (MHM) method is a member of the family of multiscale methods. Initially proposed for the Darcy model in [25], the MHM method yields superconvergent solutions and provides a face-based a posteriori error estimates which drives adaptativity (c.f. [6]). The MHM method is a byproduct of the primal hybridization of the original weak formulation analyzed in [35]. The strategy consists of characterizing, first, the exact solution of the hybrid formulation as the solution of an equivalent local-global problem. Then, discretization uncouples local and global problems making the (independent) local problems responsible for the computation of the multiscale base functions. The global problem, defined on the skeleton

Key words and phrases. A posteriori error estimator; Stokes operator; multiscale finite element; high-order approximation.

The first author was partially supported by CONICYT-Chile through project FONDECYT-1150174 and project AFB170001 of the PIA Program: Concurso Apoyo a Centros Científicos y Tecnológicos de Excelencia con Financiamiento Basal.

The second author was partially supported by CONICYT-Chile through scholarship PCHA/Doctorado Nacional and project AFB170001 of the PIA Program: Concurso Apoyo a Centros Científicos y Tecnológicos de Excelencia con Financiamiento Basal.

The third author was partially supported by CNPq/Brazil No. 301576/2013-0, EU H2020 Program and from MCTI/RNPBrazil under the HPC4E Project, Grant Agreement No. 689772, and by CAPES/Brazil No. 88881.143295/2017-01 under the PHOTOM Project. 
of the coarse partition, responds for the computations of degrees of freedom. Also, the MHM method can be used to approximate the solution of singularly perturbed reactive-advective-diffusive problems [26], linear elasticity [24] and Maxwell equations [31]. It is important to mention that the hybridization strategy has also been used at the discrete level to develop domain decomposition methods (c.f. $[3,13,12]$ ).

This work proposes a new residual a posteriori error estimator for the MHM method applied to the Stokes/Brinkman problem. It accounts for the multi-level numerical approximation of the method presented in [7]. Indeed, the error indicator has two-levels of contributions: $\eta_{1}$ related to the jump of the discrete velocity on the skeleton of the first-level mesh, similar to those that appear in previous works (see [6, 24], for instance), and $\eta_{2}$ coming from the approximation error of the solutions at the local problems. The proofs of efficiency and reliability of $\eta_{1}$ follow closely the strategy presented in [6] and [24] for the transport and elasticity equations, respectively. Here, we extend it to cope with mixed Stokes/Brinkman problems. The proof of the efficiency of $\eta_{2}$ depends on the choice of the second-level solver. Here, we chose the stabilized finite element method USFEM [10], which allow equal-order interpolations for the pressure and velocity, and then, we revisit the proof of efficiency proposed in the classic work [37] in the context of the MHM methodology. As a result, we prove that the new multiscale a posteriori error estimator is locally efficient and reliable. Also, we use the two-level information of the error estimator to drive skeleton mesh refinement and to select appropriate independent second-level meshes. The latter is fundamental to secure MHM's super-convergence. The proposed a posteriori error estimator is, up to our knowledge, the first for the MHM method to leverage the approximation error coming from the second-level solutions.

The paper is organized as follows: Section 2 introduces the model problem, and Section 3 revisits the one and two-level MHM methods proposed in [7], and gives some preliminary results. Section 4 presents the multiscale a posteriori error estimator and its analysis. Also, it outlines two adaptive algorithms based on the multi-level structure of the estimator. Three examples assess the theoretical results in Section 5, and conclusions are in Section 6. The appendix section contains some intermediary theoretical results.

\section{Model Problem And Preliminaries}

Let $\Omega \subset \mathbb{R}^{d}, d \in\{2,3\}$, be an open and bounded polytopal domain with boundary $\partial \Omega$. The generalized Stokes problem, also called Brinkman problem, consists of finding a velocity $\boldsymbol{u}: \Omega \rightarrow \mathbb{R}^{d}$ and a pressure $p: \Omega \rightarrow \mathbb{R}$ fields such that

$$
\begin{aligned}
-\nu \Delta \boldsymbol{u}+\gamma \boldsymbol{u}+\nabla p & =\boldsymbol{f} & & \text { in } \Omega, \\
\nabla \cdot \boldsymbol{u} & =0 & & \text { in } \Omega, \\
\boldsymbol{u} & =\boldsymbol{g} & & \text { on } \partial \Omega .
\end{aligned}
$$

Here $\nu \in \mathbb{R}^{+}$is the diffusion coefficient, $\gamma \in L^{\infty}(\Omega)^{d \times d}$ is a tensor which may contains multiscale features of the media, $\boldsymbol{f} \in L^{2}(\Omega)^{d}$ is a body force and the function $\boldsymbol{g} \in H^{1 / 2}(\partial \Omega)^{d}$ satisfies the compatibility condition $\int_{\partial \Omega} \boldsymbol{g} \cdot \boldsymbol{n} \mathrm{d} s=0$, where $\boldsymbol{n}$ is the outward unit normal vector of $\partial \Omega$.

Also, we assume $\gamma$ is a symmetric and definite positive tensor which is uniformly elliptic, i.e., there exist constants $\gamma_{\min }, \gamma_{\max }>0$ such that

$$
\gamma_{\min }|\boldsymbol{\xi}|^{2} \leq \boldsymbol{\xi}^{T} \boldsymbol{\gamma}(\boldsymbol{x}) \boldsymbol{\xi} \leq \gamma_{\max }|\boldsymbol{\xi}|^{2}, \quad \forall \boldsymbol{\xi} \in \mathbb{R}^{d}, \forall \boldsymbol{x} \in \Omega,
$$

where $|\cdot|$ is the Euclidean norm. Above and hereafter the Sobolev spaces have their usual meaning.

2.1. Notations. The standard variational mixed formulation of problem (2.1) reads: Find $\boldsymbol{u} \in H^{1}(\Omega)^{d}$, with $\boldsymbol{u}=\boldsymbol{g}$ on $\partial \Omega$, and $p \in L_{0}^{2}(\Omega)$ such that

$$
\begin{aligned}
a(\boldsymbol{u}, \boldsymbol{v})+b(\boldsymbol{v}, p) & =(\boldsymbol{f}, \boldsymbol{v})_{\Omega} \quad \text { for all } \boldsymbol{v} \in H_{0}^{1}(\Omega)^{d}, \\
b(\boldsymbol{u}, q) & =0 \quad \text { for all } q \in L_{0}^{2}(\Omega),
\end{aligned}
$$

where $L_{0}^{2}(\Omega)$ stands for the space of functions in $L^{2}(\Omega)$ with zero mean value in $\Omega$, and the bilinear forms $a(\cdot, \cdot)$ and $b(\cdot, \cdot)$ are defined by

$$
a(\boldsymbol{w}, \boldsymbol{v}):=(\nu \nabla \boldsymbol{w}, \nabla \boldsymbol{v})_{\Omega}+(\boldsymbol{\gamma} \boldsymbol{w}, \boldsymbol{v})_{\Omega} \quad \text { and } \quad b(\boldsymbol{v}, q):=-(\nabla \cdot \boldsymbol{v}, q)_{\Omega},
$$

for all $\boldsymbol{w}, \boldsymbol{v} \in H^{1}(\Omega)^{d}$ and $q \in L_{0}^{2}(\Omega)$. As usual, $(\cdot, \cdot)_{D}$ denotes the $L^{2}$ inner product on a open set $D$ (we don't make a distinction between vector-valued and scalar-valued functions). Problem (2.3) is well-posed (see for instance [21]). 
Let $\left\{\mathcal{T}_{H}\right\}_{H>0}$ be a family of shape-regular meshes of $\bar{\Omega}$, composed of simplex $K$ of diameter $H_{K}$, and of global mesh size $H:=\max \left\{H_{K}: K \in \mathcal{T}_{H}\right\}$. Hereafter we shall use the terminology usually employed for three-dimensional domains, with the restriction to two-dimensional problems being straightforward. As such, each element $K$ has a boundary $\partial K$ consisting of faces $F$, with diameter $H_{F}$. The collection of all faces $F$, in a triangulation $\mathcal{T}_{H}$, is denoted by $\mathcal{E}_{H}$, which is decomposed into the set of internal faces $\mathcal{E}_{0}$ and the set of faces on the Dirichlet boundary $\mathcal{E}_{D}$. We denote by $\mathcal{T}_{\tilde{H}}(F)$ a triangulation of $F \in \mathcal{E}_{H}$, where $\tilde{H}$ is the maximum size of all $\tilde{F}$ belonging to $\mathcal{T}_{\tilde{H}}(F)$. For each $K \in \mathcal{T}_{H}$, we denote by $\boldsymbol{n}^{K}$ the outward normal on $\partial K$, and let $\boldsymbol{n}_{F}^{K}:=\left.\boldsymbol{n}^{K}\right|_{F}$ for each $F \subset \partial K$.

On top of a mesh $\mathcal{T}_{H}$, we define the following spaces

$$
\begin{aligned}
& \mathbf{V}:=H^{1}\left(\mathcal{T}_{H}\right)^{d}:=\left\{\boldsymbol{v} \in L^{2}(\Omega)^{d}:\left.\boldsymbol{v}\right|_{K} \in H^{1}(K)^{d} \quad \text { for all } K \in \mathcal{T}_{H}\right\}, \\
& \boldsymbol{\Lambda}:=\left\{\left.\boldsymbol{\sigma} \boldsymbol{n}^{K}\right|_{\partial K} \in H^{-1 / 2}(\partial K)^{d} \quad \text { for all } K \in \mathcal{T}_{H}: \boldsymbol{\sigma} \in H(\operatorname{div} ; \Omega)\right\},
\end{aligned}
$$

and $Q:=L^{2}(\Omega)$. We define an inner product on $\mathbf{V}$ by

$$
(\boldsymbol{u}, \boldsymbol{v})_{\mathbf{V}}:=\frac{1}{d_{\Omega}^{2}}(\boldsymbol{u}, \boldsymbol{v})_{\Omega}+\sum_{K \in \mathcal{T}_{H}}(\nabla \boldsymbol{u}, \nabla \boldsymbol{v})_{K} \quad \text { for all } \boldsymbol{u}, \boldsymbol{v} \in \mathbf{V}
$$

where $d_{\Omega}$ is the diameter of $\Omega$. We equip the spaces $H(\operatorname{div} ; \Omega), \mathbf{V}$ and $\mathbf{V} \times Q$ with the norms,

$$
\begin{aligned}
\|\boldsymbol{\sigma}\|_{\text {div }, \Omega}^{2} & :=\sum_{K \in \mathcal{T}_{H}}\left(\|\boldsymbol{\sigma}\|_{0, K}^{2}+d_{\Omega}^{2}\|\nabla \cdot \boldsymbol{\sigma}\|_{0, K}^{2}\right), \\
\|\boldsymbol{v}\|_{\mathbf{V}}^{2} & :=\sum_{K \in \mathcal{T}_{H}}\left(d_{\Omega}^{-2}\|\boldsymbol{v}\|_{0, K}^{2}+\|\nabla \boldsymbol{v}\|_{0, K}^{2}\right),
\end{aligned}
$$

and

$$
\|(\boldsymbol{v}, q)\|_{\mathbf{V} \times Q}^{2}:=\|\boldsymbol{v}\|_{\mathbf{V}}^{2}+\|q\|_{Q}^{2}
$$

respectively, and the space $\boldsymbol{\Lambda}$ with the quotient norm

$$
\|\boldsymbol{\mu}\|_{\boldsymbol{\Lambda}}:=\inf _{\substack{\boldsymbol{\sigma} \in H(\operatorname{div} ; \Omega) \\ \boldsymbol{\sigma} \boldsymbol{n}^{K}=\boldsymbol{\mu} \text { on } \partial K, K \in \mathcal{T}_{H}}}\|\boldsymbol{\sigma}\|_{\operatorname{div}, \Omega} .
$$

We denote by $\langle\cdot, \cdot\rangle_{\partial D}$ the dual pairing between $H^{-1 / 2}(\partial D)^{d}$ and $H^{1 / 2}(\partial D)^{d}$ for $D \subseteq \Omega$ and

$$
(\boldsymbol{\mu}, \boldsymbol{v})_{\partial \mathcal{T}_{H}}:=\sum_{K \in \mathcal{T}_{H}}\langle\boldsymbol{\mu}, \boldsymbol{v}\rangle_{\partial K},
$$

and the following norms in $H^{-1 / 2}(\partial D)^{d}$ and $H^{1 / 2}(\partial D)^{d}$, respectively,

$$
\|\boldsymbol{\mu}\|_{-1 / 2, \partial D}:=\sup _{\boldsymbol{v} \in H^{1 / 2}(\partial D)^{d}} \frac{\langle\boldsymbol{\mu}, \boldsymbol{v}\rangle_{\partial D}}{\|\boldsymbol{v}\|_{1 / 2, \partial D}} \text { and }\|\boldsymbol{v}\|_{1 / 2, \partial D}:=\inf _{\substack{\mathbf{w} \in H^{1}(D)^{d} \\ \mathbf{w}=\boldsymbol{v} \text { on } \partial D}}\|\mathbf{w}\|_{1, D} .
$$

We recall from [6, Lemma 8.3] that the norm (2.10) is equivalent to a dual norm, namely,

$$
\frac{\sqrt{2}}{2}\|\boldsymbol{\mu}\|_{\boldsymbol{\Lambda}} \leq \sup _{\boldsymbol{v} \in \mathbf{V}} \frac{(\boldsymbol{\mu}, \boldsymbol{v})_{\partial \mathcal{T}_{H}}}{\|\boldsymbol{v}\|_{\mathbf{V}}} \leq\|\boldsymbol{\mu}\|_{\boldsymbol{\Lambda}} \quad \text { for all } \boldsymbol{\mu} \in \boldsymbol{\Lambda} \text {. }
$$

Above and hereafter we lighten notation and understand the supremum to be taken over sets excluding the zero function, even though this is not specifically indicated.

Next, following [6], we replace (2.3) by the following hybrid formulation: Find $(\boldsymbol{u}, p, \boldsymbol{\lambda}, \rho) \in \mathbf{V} \times Q \times \boldsymbol{\Lambda} \times \mathbb{R}$ such that

$$
\left\{\begin{aligned}
a(\boldsymbol{u}, \boldsymbol{v})+b(\boldsymbol{v}, p)+(\boldsymbol{\lambda}, \boldsymbol{v})_{\partial \mathcal{T}_{H}} & =(\boldsymbol{f}, \boldsymbol{v})_{\mathcal{T}_{H}} \text { for all } \boldsymbol{v} \in \mathbf{V} \\
b(\boldsymbol{u}, q)+(\rho, q)_{\Omega} & =0 \quad \text { for all } q \in Q \\
(\boldsymbol{\mu}, \boldsymbol{u})_{\partial \mathcal{T}_{H}} & =\langle\boldsymbol{\mu}, \boldsymbol{g}\rangle_{\partial \Omega} \text { for all } \boldsymbol{\mu} \in \boldsymbol{\Lambda} \\
(\xi, p)_{\Omega} & =0 \quad \text { for all } \xi \in \mathbb{R}
\end{aligned}\right.
$$


and we found that $(\boldsymbol{u}, p)$ solution of (2.13) coincides with the solution of (2.3) as shown in [7, Theorem 6]. Above and hereafter, we surcharge the notation $a(\cdot, \cdot)$ and $b(\cdot, \cdot)$ to extend them to the space $\mathbf{V}$ as follows

$$
a(\boldsymbol{w}, \boldsymbol{v}):=\sum_{K \in \mathcal{T}_{H}} a_{K}(\boldsymbol{w}, \boldsymbol{v}) \quad \text { with } \quad a_{K}(\boldsymbol{w}, \boldsymbol{v}):=(\nu \nabla \boldsymbol{w}, \nabla \boldsymbol{v})_{K}+(\boldsymbol{\gamma} \boldsymbol{w}, \boldsymbol{v})_{K},
$$

and

$$
b(\boldsymbol{v}, q):=\sum_{K \in \mathcal{T}_{H}} b_{K}(\boldsymbol{v}, q) \quad \text { with } \quad b_{K}(\boldsymbol{v}, q):=-(\nabla \cdot \boldsymbol{v}, q)_{K},
$$

for all $\boldsymbol{w}, \boldsymbol{v} \in \mathbf{V}$ and $q \in Q$.

2.2. Preliminar results. Let $\mathbf{V}_{0}$ be the closed subspace of the Hilbert space $\mathbf{V}$ defined by

$$
\mathbf{V}_{0}:=\{\boldsymbol{v} \in \mathbf{V}: a(\boldsymbol{v}, \boldsymbol{w})=0 \text { for all } \boldsymbol{w} \in \mathbf{V}\} .
$$

Observe that for the Stokes problem $(\boldsymbol{\gamma}=\mathbf{0})$

$$
\mathbf{V}_{0}=\left\{\boldsymbol{v} \in \mathbf{V}:\left.\boldsymbol{v}\right|_{K} \in \mathbb{P}_{0}(K)^{d} \text { for all } K \in \mathcal{T}_{H}\right\},
$$

where $\mathbb{P}_{0}(K)$ stands for the space of constant polynomials defined on $K$, and $\mathbf{V}_{0}=\{\mathbf{0}\}$ otherwise. As such, we can decompose $\mathbf{V}$ as follows

$$
\mathbf{V}=\mathbf{V}_{0} \oplus \mathbf{V}_{0}^{\perp}
$$

where $\mathbf{V}_{0}^{\perp}$ is the orthogonal complement with respect to the inner-product $(\cdot, \cdot) \mathbf{V}$. Note that for the Stokes equations $(\boldsymbol{\gamma}=\mathbf{0})$, it is given by

$$
\mathbf{V}_{0}^{\perp}=\left\{\boldsymbol{v} \in \mathbf{V}:\left.\boldsymbol{v}\right|_{K} \in\left[H^{1}(K) \cap L_{0}^{2}(K)\right]^{d} \text { for all } K \in \mathcal{T}_{H}\right\},
$$

and $\mathbf{V}_{0}^{\perp}=\mathbf{V}$ otherwise. We shall denote by $\mathbf{V}_{0}^{\perp}(K), \mathbf{V}(K)$ and $Q(K)$ the spaces of vector functions in $\mathbf{V}_{0}^{\perp}$, $\mathbf{V}$ and $Q$ restricted to $K$, respectively. Also, the corresponding restricted norms are denoted by $\|\cdot\|_{\mathbf{V}(K)}$ and $\|\cdot\|_{Q(K)}$, and the local product norm by $\|\cdot, \cdot\|_{\mathbf{V}(K) \times Q(K)}$.

The next results shall be intensively used in the rest of this work. Although being a direct consequence of standard saddle-point theory, they may not be easy to find in the literature, and then, we decided to detail them here.

Theorem 2.1. There exists a constant $\alpha>0$, independent of $K$, such that

$$
\sup _{\boldsymbol{v} \in \mathbf{V}_{0}(K)} \frac{(\nabla \cdot \boldsymbol{v}, q)_{K}}{\|\boldsymbol{v}\|_{\mathbf{V}(K)}} \geq \alpha\|q\|_{Q(K)}
$$

for all $q \in Q(K)$.

Proof. We first consider the Stokes case, that is,

$$
\mathbf{V}_{0}(K)^{\perp}=\left[H^{1}(K) \cap L_{0}^{2}(K)\right]^{d} .
$$

Let $q \in Q(K)$. Thanks to the decomposition $L^{2}(K)=L_{0}^{2}(K) \oplus \mathbb{P}_{0}(K)$, it holds $q=\tilde{q}+q_{0}$ with $\tilde{q} \in L_{0}^{2}(K)$ and $q_{0}=|K|^{-1} \int_{K} q \mathrm{~d} x$. Let $\tilde{\boldsymbol{w}} \in H_{0}^{1}(K)^{d}$ be such that

$$
\nabla \cdot \tilde{\boldsymbol{w}}=\tilde{q} \quad \text { in } K
$$

satisfying

$$
|\tilde{\boldsymbol{w}}|_{1, K} \leq C_{d} \frac{H_{K}}{\rho_{K}}\|\tilde{q}\|_{0, K},
$$

where $\rho_{K}$ is the radius of the ball inscribed in $K$ and $C_{d}$ is a positive constant which depends only on $d$. The existence of such a function $\tilde{\boldsymbol{w}}$ is ensured in [17, Theorem 5.2]. Since we supposed that $\left\{\mathcal{T}_{H}\right\}_{H>0}$ is shape-regular, there exists $\sigma_{0}>0$ such that

$$
\frac{H_{K}}{\rho_{K}} \leq \sigma_{0}
$$

for all $K \in \mathcal{T}_{H}$ and all $H_{K}$, thus from (2.16) we have

$$
|\tilde{\boldsymbol{w}}|_{1, K} \leq C_{d} \sigma_{0}\|\tilde{q}\|_{0, K}
$$

Setting

$$
\boldsymbol{w}:=\tilde{\boldsymbol{w}}+\frac{q_{0}}{d} \boldsymbol{x}-\frac{1}{|K|} \int_{K}\left(\tilde{\boldsymbol{w}}+\frac{q_{0}}{d} \boldsymbol{x}\right) \mathrm{d} x,
$$


from $(2.15)$ we have that

$$
\nabla \cdot \boldsymbol{w}=q \quad \text { in } K
$$

and from $(2.17)$

$$
\begin{aligned}
|\boldsymbol{w}|_{1, K} & \leq|\tilde{\boldsymbol{w}}|_{1, K}+\left|d^{-1} q_{0} \boldsymbol{x}\right|_{1, K} \\
& \leq C_{d} \sigma_{0}\|\tilde{q}\|_{0, K}+d^{-1 / 2}\left\|q_{0}\right\|_{0, K} \\
& \leq \max \left\{C_{d} \sigma_{0}, d^{-1 / 2}\right\}\|q\|_{0, K} .
\end{aligned}
$$

In addition, since $\boldsymbol{w} \in\left[H^{1}(K) \cap L_{0}^{2}(K)\right]^{d}$, from [11, Theorem 3.2], we have

$$
\|\boldsymbol{w}\|_{0, K} \leq \frac{H_{K}}{\pi}|\boldsymbol{w}|_{1, K}
$$

Then, from (2.18), (2.19) and (2.20), we get

$$
\begin{aligned}
\sup _{\boldsymbol{v} \in\left[H^{1}(K) \cap L_{0}^{2}(K)\right]^{d}} \frac{(\nabla \cdot \boldsymbol{v}, q)_{K}}{\|\boldsymbol{v}\|_{\mathbf{V}(K)}} & \geq \frac{(\nabla \cdot \boldsymbol{w}, q)_{K}}{\left(d_{\Omega}^{-2}\|\boldsymbol{w}\|_{0, K}^{2}+|\boldsymbol{w}|_{1, K}^{2}\right)^{1 / 2}} \\
& \geq\left(\left(\frac{1}{\pi^{2}}+1\right)^{1 / 2} \max \left\{C_{d} \sigma_{0}, d^{-1 / 2}\right\}\right)^{-1}\|q\|_{0, K} .
\end{aligned}
$$

For the Brinkman case, that is $\mathbf{V}_{0}(K)^{\perp}=H^{1}(K)^{d}$, we use (2.21) to obtain

$$
\begin{aligned}
\sup _{\boldsymbol{v} \in H^{1}(K)^{d}} \frac{(\nabla \cdot \boldsymbol{v}, q)_{K}}{\|\boldsymbol{v}\|_{\mathbf{V}(K)}} & \geq \sup _{\boldsymbol{v} \in\left[H^{1}(K) \cap L_{0}^{2}(K)\right]^{d}} \frac{(\nabla \cdot \boldsymbol{v}, q)_{K}}{\|\boldsymbol{v}\|_{\mathbf{V}(K)}} \\
& \geq\left(\left(\frac{1}{\pi^{2}}+1\right)^{1 / 2} \max \left\{C_{d} \sigma_{0}, d^{-1 / 2}\right\}\right)^{-1}\|q\|_{0, K}
\end{aligned}
$$

ending the proof.

Theorem 2.2. There exists a constant $\beta>0$, independent of $H_{K}$, such that

$$
\sup _{(\boldsymbol{v}, q) \in \mathbf{V}_{0}(K)^{\perp} \times Q(K)} \frac{a_{K}(\boldsymbol{w}, \boldsymbol{v})+b_{K}(\boldsymbol{v}, r)-b_{K}(\boldsymbol{w}, q)}{\|(\boldsymbol{v}, q)\|_{\mathbf{V}(K) \times Q(K)}} \geq \beta\|(\boldsymbol{w}, r)\|_{\mathbf{V}(K) \times Q(K)}
$$

for all $(\boldsymbol{w}, r) \in \mathbf{V}_{0}(K)^{\perp} \times Q(K)$.

Proof. The result follows from the ellipticity of the bilinear form $a_{K}(\cdot, \cdot)$ and Theorem 2.1.

Remark 1. Using Theorem 2.2 and Riesz Representation Theorem, the following global inf-sup condition holds

$$
\begin{aligned}
& \sup _{(\boldsymbol{v}, q) \in \mathbf{V}_{0}^{\perp} \times Q} \frac{a(\boldsymbol{w}, \boldsymbol{v})+b(\boldsymbol{v}, r)-b(\boldsymbol{w}, q)}{\|(\boldsymbol{v}, q)\|_{\mathbf{V} \times Q}}=\left\{\sum_{K \in \mathcal{T}_{H}}\left(\sup _{(\boldsymbol{v}, q) \in \mathbf{V}_{0}(K)^{\perp} \times Q(K)} \frac{a_{K}(\boldsymbol{w}, \boldsymbol{v})+b_{K}(\boldsymbol{v}, r)-b_{K}(\boldsymbol{w}, q)}{\|(\boldsymbol{v}, q)\|_{\mathbf{V}(K) \times Q(K)}}\right)^{2}\right\}^{1 / 2} \\
& \geq \beta\|(\boldsymbol{w}, r)\|_{\mathbf{V} \times Q}
\end{aligned}
$$

for all $(\boldsymbol{w}, r) \in \mathbf{V}_{0}^{\perp} \times Q$, where $\beta>0$ is independent of $H$.

Let us now adresse the second-level discretization. To this end, we denote by $\left\{\mathcal{T}_{h}^{K}\right\}_{h>0}$ a regular family of meshes of $K$, where $h$ is the characteristic length of $\mathcal{T}_{h}^{K}$. We notice that the family $\left\{\mathcal{T}_{h}^{K}\right\}_{h>0}$ may differ in each $K \in \mathcal{T}_{H}$. Also, we denote by $\mathcal{E}_{h}^{K}$ the set of faces on $\mathcal{T}_{h}^{K}$, and by $\mathcal{E}_{0}^{K}$ the set of internal faces. To each $\zeta \in \mathcal{E}_{h}^{K}$ we associate a normal vector $\boldsymbol{n}_{\zeta}^{\tau}$, taking care to ensure this is facing outward on $\partial K$.

Associated to the second-level meshes for each $K \in \mathcal{T}_{H}$, we define the standard polynomial bubble functions which shall be used in the proofs. Specifically, let $b_{\tau}^{K}$ and $b_{\zeta}^{K}$ be the bubble functions with support in $\tau \in \mathcal{T}_{h}^{K}$ and $\omega_{\zeta} \in \mathcal{E}_{h}^{K}$, respectively, and defined with respect to the barycentric coordinates (see for instance [4, Section 2.3.1] for details). For the sake of completeness, we line up two theorems that summarize the 
main properties of these functions, observing that their vector versions counterpart are straightforward. For this, we adopt in the rest of the paper the following notation

$$
\begin{aligned}
& a \preceq b \Longleftrightarrow a \leq C b, \\
& a \simeq b \Longleftrightarrow a \preceq b \text { and } b \preceq a,
\end{aligned}
$$

where the positive constant $C$ is independent of $H$ and $h$, and $\mathbb{P}_{n}\left(\mathcal{T}_{h}^{K}\right)$ is the space of piecewise polynomial functions of degree equal or less than $n$ in each element $\tau \in \mathcal{T}_{h}^{K}$.

Theorem 2.3. Let $K \in \mathcal{T}_{H}$ and let $b_{\tau}^{K}$ be the element bubble function corresponding to $\tau \in \mathcal{T}_{h}^{K}$. Then

$$
\left\|v_{h}\right\|_{0, \tau}^{2} \preceq\left(b_{\tau}^{K} v_{h}, v_{h}\right)_{\tau} \preceq\left\|v_{h}\right\|_{0, \tau}^{2}
$$

and

$$
\left\|v_{h}\right\|_{0, \tau} \preceq\left\|b_{\tau}^{K} v_{h}\right\|_{0, \tau}+h_{\tau}\left|b_{\tau}^{K} v_{h}\right|_{1, \tau} \preceq\left\|v_{h}\right\|_{0, \tau}
$$

for all $v_{h} \in \mathbb{P}_{n}\left(\mathcal{T}_{h}^{K}\right), n \geq 0$.

Proof. See [4, Theorem 2.2].

Theorem 2.4. Let $K \in \mathcal{T}_{H}$ and let $b_{\zeta}^{K}$ be the corresponding face bubble function on face $\zeta \in \mathcal{E}_{h}^{K}$ and $\tau \in \mathcal{T}_{h}^{K}$ such that $\tau$ is in the support of $b_{\zeta}^{K}$. Then

$$
\left\|v_{h}\right\|_{0, \zeta}^{2} \preceq\left(b_{\zeta}^{K} v_{h}, v_{h}\right)_{\zeta} \preceq\left\|v_{h}\right\|_{0, \zeta}^{2}
$$

and

$$
h_{\tau}^{-1 / 2}\left\|b_{\zeta}^{K} v_{h}\right\|_{0, \tau}+h_{\tau}^{1 / 2}\left|b_{\zeta}^{K} v_{h}\right|_{1, \tau} \preceq\left\|v_{h}\right\|_{0, \zeta}
$$

for all $v_{h} \in \mathbb{P}_{n}\left(\mathcal{T}_{h}^{K}\right), n \geq 0$

Proof. See [4, Theorem 2.4].

Also, let $\mathscr{C}_{h}^{K}: H^{1}(K) \rightarrow V_{1}^{K}$ be the Clément interpolation operator defined on each $K \in \mathcal{T}_{H}$, where $V_{1}^{K}$ is given by

$$
V_{1}^{K}:=\left\{v_{h} \in C^{0}(K): v_{h} \in \mathbb{P}_{1}(\tau) \text { for all } \tau \in \mathcal{T}_{h}^{K}\right\}
$$

It verifies the following local stability and approximation properties (cf. [16] and [19]):

$$
\begin{aligned}
\left\|\mathscr{C}_{h}(v)\right\|_{0, \tau} \preceq\|v\|_{0, \tilde{\omega}_{\tau}} \quad \forall \tau \in \mathcal{T}_{h}^{K}, \\
\left\|v-\mathscr{C}_{h}(v)\right\|_{0, \tau} \preceq h_{\tau}|v|_{1, \tilde{\omega}_{\tau}} \quad \forall \tau \in \mathcal{T}_{h}^{K}, \\
\left\|v-\mathscr{C}_{h}(v)\right\|_{0, \zeta} \preceq h_{\zeta}^{1 / 2}|v|_{1, \tilde{\omega}_{\zeta}} \quad \forall \zeta \in \mathcal{E}_{h}^{K},
\end{aligned}
$$

for all $v \in H^{1}(K)$, where $\tilde{\omega}_{\tau}$ and $\tilde{\omega}_{\zeta}$ are the set of elements $\tau \in \mathcal{T}_{h}^{K}$ sharing at least one vertex with $\tau$ and $\zeta$, respectively. Furthermore, we denote by $\mathscr{C}_{h}^{K}(\boldsymbol{v})$ the vector function whose components are the Clément interpolation of each component of $\boldsymbol{v} \in H^{1}(K)^{d}$.

\section{The MHM Method}

This section summarizes the main ideas of the MHM method proposed in [7]. The basic idea behind the MHM approach is to leverage the nature of hybrid problem (2.13), and characterize the exact solution regarding the well-posed independent local problems and a face-based global problem which ties everything together. The one- and two-level MHM method results from the discretization of the global (one-level) and local (two-level) problems, respectively. 
3.1. Characterizing the exact solution. Let us consider the following local bilinear form $B_{K}:\left(\mathbf{V}_{0}^{\perp}(K) \times\right.$ $Q(K)) \times\left(\mathbf{V}_{0}^{\perp}(K) \times Q(K)\right)$ defined by

$$
B_{K}((\boldsymbol{w}, r),(\boldsymbol{v}, q)):=a_{K}(\boldsymbol{w}, \boldsymbol{v})+b_{K}(\boldsymbol{v}, r)-b_{K}(\boldsymbol{w}, q)
$$

for all $(\boldsymbol{w}, r),(\boldsymbol{v}, q) \in \mathbf{V}_{0}^{\perp}(K) \times Q(K)$.

We define the following linear mappings:

- $\boldsymbol{T}: \boldsymbol{\Lambda} \rightarrow \mathbf{V}_{0}^{\perp} \times Q$ such that $\left.\boldsymbol{T} \boldsymbol{\mu}\right|_{K}:=\left(T^{\boldsymbol{u}} \boldsymbol{\mu}, T^{p} \boldsymbol{\mu}\right)$ solves

$$
B_{K}\left(\left(T^{\boldsymbol{u}} \boldsymbol{\mu}, T^{p} \boldsymbol{\mu}\right),(\boldsymbol{v}, q)\right)=-\langle\boldsymbol{\mu}, \boldsymbol{v}\rangle_{\partial K} \quad \text { for all }(\boldsymbol{v}, q) \in \mathbf{V}_{0}^{\perp}(K) \times Q(K) ;
$$

- $\hat{\boldsymbol{T}}: L^{2}(\Omega)^{d} \rightarrow \mathbf{V}_{0}^{\perp} \times Q$ such that $\left.\hat{\boldsymbol{T}} \boldsymbol{r}\right|_{K}:=\left(\hat{T}^{\boldsymbol{u}} \boldsymbol{r}, \hat{T}^{p} \boldsymbol{r}\right)$ solves

$$
B_{K}\left(\left(\hat{T}^{\boldsymbol{u}} \boldsymbol{r}, \hat{T}^{p} \boldsymbol{r}\right),(\boldsymbol{v}, q)\right)=(\boldsymbol{r}, \boldsymbol{v})_{K} \quad \text { for all }(\boldsymbol{v}, q) \in \mathbf{V}_{0}^{\perp}(K) \times Q(K) ;
$$

- $\overline{\boldsymbol{T}}: \mathbb{R} \rightarrow \mathbf{V}_{0}^{\perp} \times Q$ such that $\left.\overline{\boldsymbol{T}} \xi\right|_{K}:=\left(\bar{T}^{u} \xi, \bar{T}^{p} \xi\right)$ solves

$$
B_{K}\left(\left(\bar{T}^{\boldsymbol{u}} \xi, \bar{T}^{p} \xi\right),(\boldsymbol{v}, q)\right)=-(\xi, q)_{K} \quad \text { for all }(\boldsymbol{v}, q) \in \mathbf{V}_{0}^{\perp}(K) \times Q(K) .
$$

Owing to decomposition (2.14), and noting that $a(\boldsymbol{w}, \boldsymbol{v})=0$ when $\boldsymbol{w} \in \mathbf{V}_{0}$ or $\boldsymbol{v} \in \mathbf{V}_{0}$, we rewrite (2.13) as the following global-local problem: Find $\left(\boldsymbol{u}_{0}, \boldsymbol{\lambda}, \rho\right) \in \mathbf{V}_{0} \times \boldsymbol{\Lambda} \times \mathbb{R}$ such that

$$
\left\{\begin{aligned}
\left(\boldsymbol{\lambda}, \boldsymbol{v}_{0}\right)_{\partial \mathcal{T}_{H}} & =\left(\boldsymbol{f}, \boldsymbol{v}_{0}\right)_{\mathcal{T}_{H}}, \\
\left(\boldsymbol{\mu}, \boldsymbol{u}_{0}\right)_{\partial \mathcal{T}_{H}}+\left(\boldsymbol{\mu}, T^{\boldsymbol{u}} \boldsymbol{\lambda}\right)_{\partial \mathcal{T}_{H}}+\left(\boldsymbol{\mu}, \bar{T}^{\boldsymbol{u}} \rho\right)_{\partial \mathcal{T}_{H}} & =\langle\boldsymbol{\mu}, \boldsymbol{g}\rangle_{\partial \Omega}-\left(\boldsymbol{\mu}, \hat{T}^{u} \boldsymbol{f}\right)_{\partial \mathcal{T}_{H}}, \\
\left(\xi, T^{p} \boldsymbol{\lambda}\right)_{\Omega}+\left(\xi, \bar{T}^{p} \rho\right)_{\Omega} & =-\left(\xi, \hat{T}^{p} \boldsymbol{f}\right)_{\Omega},
\end{aligned}\right.
$$

for all $\left(\boldsymbol{v}_{0}, \boldsymbol{\mu}, \xi\right) \in \mathbf{V}_{0} \times \boldsymbol{\Lambda} \times \mathbb{R}$.

Hereafter, we assume that the global problem (3.5) is well-posed. Hence, from the linearity of problems (3.2)-(3.4), we find that the exact $\boldsymbol{u}$ and $p$ can be characterized as follows

$$
\boldsymbol{u}=\boldsymbol{u}_{0}+T^{\boldsymbol{u}} \boldsymbol{\lambda}+\hat{T}^{\boldsymbol{u}} \boldsymbol{f} \text { and } p=T^{p} \boldsymbol{\lambda}+\hat{T}^{p} \boldsymbol{f}
$$

where we used (see [7]) that $\rho=0$. Next, we prove that operators $\boldsymbol{T}, \hat{\boldsymbol{T}}$ and $\overline{\boldsymbol{T}}$ are stable with respect to the given data.

Theorem 3.1. The operators $\boldsymbol{T}, \hat{\boldsymbol{T}}$ and $\overline{\boldsymbol{T}}$, given by (3.2), (3.3) and (3.4), respectively, are well-defined. Moreover, we have that

$$
\begin{aligned}
\|\boldsymbol{T} \boldsymbol{\mu}\|_{\mathbf{V} \times Q} & \preceq\|\boldsymbol{\mu}\|_{\boldsymbol{\Lambda}} \quad \text { for all } \boldsymbol{\mu} \in \boldsymbol{\Lambda} \\
\|\hat{\boldsymbol{T}} \boldsymbol{r}\|_{\mathbf{V} \times Q} & \preceq\|\boldsymbol{r}\|_{0, \Omega} \quad \text { for all } \boldsymbol{r} \in L^{2}(\Omega)^{d}, \\
\|\overline{\boldsymbol{T}} \xi\|_{\mathbf{V} \times Q} & \preceq|\xi| \quad \text { for all } \xi \in \mathbb{R} .
\end{aligned}
$$

Proof. From (2.12) and the global inf-sup (2.22) we get

$$
\begin{aligned}
\|\boldsymbol{T} \boldsymbol{\mu}\|_{\mathbf{V} \times Q} & =\left\|\left(T^{\boldsymbol{u}} \boldsymbol{\mu}, T^{p} \boldsymbol{\mu}\right)\right\|_{\mathbf{V} \times Q} \\
& \leq \frac{1}{\beta} \sup _{(\boldsymbol{v}, q) \in \mathbf{V}_{0}^{\perp} \times Q} \frac{a\left(T^{\boldsymbol{u}} \boldsymbol{\mu}, \boldsymbol{v}\right)+b\left(\boldsymbol{v}, T^{p} \boldsymbol{\mu}\right)-b\left(T^{\boldsymbol{u}} \boldsymbol{\mu}, q\right)}{\|(\boldsymbol{v}, q)\|_{\mathbf{V} \times Q}} \\
& =\frac{1}{\beta} \sup _{(\boldsymbol{v}, q) \in \mathbf{V}_{0}^{\perp} \times Q} \frac{-(\boldsymbol{\mu}, \boldsymbol{v})_{\partial \mathcal{T}_{H}}}{\|(\boldsymbol{v}, q)\|_{\mathbf{V} \times Q}} \leq \frac{1}{\beta}\|\boldsymbol{\mu}\|_{\boldsymbol{\Lambda}},
\end{aligned}
$$

where $\beta>0$ is independent of $H$. The proofs of the other two inequalities are analogous.

Note that the solution of the coupled global-local problem (3.2)-(3.5) coincides with the solution of the hybrid problem (2.13). 
3.2. The one-level MHM method. The one-level MHM method stems from the coupled problems (3.2)(3.5). We search the approximate Lagrange multipliers in the following space

$$
\boldsymbol{\Lambda}_{H}:=\left\{\boldsymbol{\lambda}_{H} \in \boldsymbol{\Lambda}:\left.\boldsymbol{\lambda}_{H}\right|_{\tilde{F}} \in \mathbb{P}_{l}(\tilde{F})^{d}, \text { for all } \tilde{F} \subset \mathcal{T}_{\tilde{H}}(F) \text {, for all } F \in \mathcal{E}_{H}\right\},
$$

where $\mathbb{P}_{l}(\tilde{F})$ stands for the space of polynomials of degree less or equal to $l \geq 0$ on $\tilde{F}$. Observe that (3.7) permits discontinuous interpolations on faces which can differ on each $F \in \mathcal{E}_{H}$. See Figure 1 for an illustration. We shall denote by $\boldsymbol{\Lambda}_{l}^{m}$ the space when $\mathcal{T}_{\tilde{H}}(F)$ is compose of $m$ elements. When $m=1$, we denote $\boldsymbol{\Lambda}_{l}^{1}$ by $\boldsymbol{\Lambda}_{l}$.
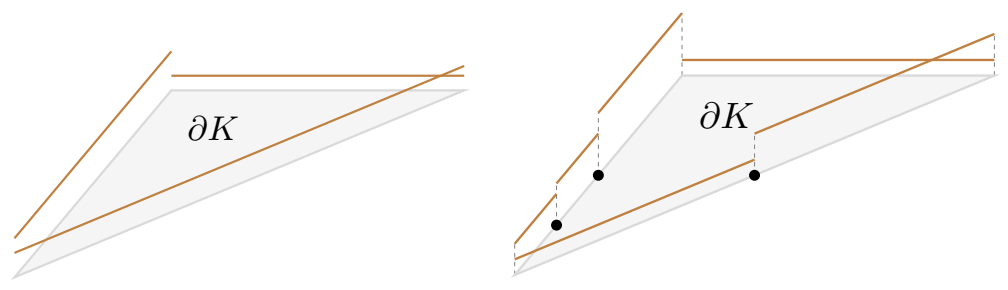

Figure 1. Two examples of $\boldsymbol{\Lambda}_{H}$ restricted to an element $K \in \mathcal{T}_{H}$.

Replacing $\boldsymbol{\Lambda}$ by $\boldsymbol{\Lambda}_{H}$ uncouples local and global problems as shown in [7]. The multiscale basis functions are computed from (3.2) with the right-hand side replaced by the polynomial base functions on faces, and the degrees of freedom are obtained from the following one-level MHM method: Find $\left(\boldsymbol{u}_{0}^{H}, \boldsymbol{\lambda}_{H}, \rho_{H}\right) \in \mathbf{V}_{0} \times \boldsymbol{\Lambda}_{H} \times \mathbb{R}$ such that

$$
\left\{\begin{array}{c}
\left(\boldsymbol{\lambda}_{H}, \boldsymbol{v}_{0}\right)_{\partial \mathcal{T}_{H}}=\left(\boldsymbol{f}, \boldsymbol{v}_{0}\right)_{\mathcal{T}_{H}}, \\
\left(\boldsymbol{\mu}_{H}, \boldsymbol{u}_{0}^{H}\right)_{\partial \mathcal{T}_{H}}+\left(\boldsymbol{\mu}_{H}, T^{\boldsymbol{u}} \boldsymbol{\lambda}_{H}\right)_{\partial \mathcal{T}_{H}}+\left(\boldsymbol{\mu}_{H}, \bar{T}^{\boldsymbol{u}} \rho_{H}\right)_{\partial \mathcal{T}_{H}}=\left\langle\boldsymbol{\mu}_{H}, \boldsymbol{g}\right\rangle_{\partial \Omega}-\left(\boldsymbol{\mu}_{H}, \hat{T}^{\boldsymbol{u}} \boldsymbol{f}\right)_{\partial \mathcal{T}_{H}}, \\
\left(\xi_{H}, T^{p} \boldsymbol{\lambda}_{H}\right)_{\Omega}+\left(\xi_{H}, \bar{T}^{p} \rho_{H}\right)_{\Omega}=-\left(\xi_{H}, \hat{T}^{p} \boldsymbol{f}\right)_{\Omega},
\end{array}\right.
$$

for all $\boldsymbol{v}_{0} \in \mathbf{V}_{0}, \boldsymbol{\mu}_{H} \in \boldsymbol{\Lambda}_{H}$ and $\xi_{H} \in \mathbb{R}$. Here $\boldsymbol{T}, \hat{\boldsymbol{T}}$ and $\overline{\boldsymbol{T}}$ are given by (3.2)-(3.4), respectively. Observe that, in the one-level method (3.8), we assume that close formulas are available for the corresponding solutions of local problems (3.2)-(3.4). Thereby, the discrete one-level approximation $\left(\boldsymbol{u}_{H}, p_{H}, \rho_{H}\right)$ of the exact solutions read

$$
\boldsymbol{u}_{H}:=\boldsymbol{u}_{0}^{H}+T^{\boldsymbol{u}} \boldsymbol{\lambda}_{H}+\hat{T}^{\boldsymbol{u}} \boldsymbol{f}+\bar{T}^{\boldsymbol{u}} \rho_{H} \quad \text { and } \quad p_{H}:=T^{p} \boldsymbol{\lambda}_{H}+\hat{T}^{p} \boldsymbol{f}+\bar{T}^{p} \rho_{H} .
$$

We remark that the one-level MHM method is non-conform in $H^{1}(\Omega)^{d}$. Nevertheless, it provides a discrete stress tensor $\boldsymbol{\sigma}_{H}$ given by

$$
\boldsymbol{\sigma}_{H}:=-\nu \nabla \boldsymbol{u}_{H}+p_{H} \mathbf{I} \quad \text { in } H(\operatorname{div} ; \Omega),
$$

since $\left.\boldsymbol{\sigma}_{H} \boldsymbol{n}^{K}\right|_{\partial K} \in \boldsymbol{\Lambda}_{H}$ for all $K \in \mathcal{T}_{H}$. Also, the discrete stress tensor $\boldsymbol{\sigma}_{H}$ is automatically in equilibrium, in each element $K \in \mathcal{T}_{H}$, i.e.,

$$
\int_{\partial K} \boldsymbol{\sigma}_{H} \boldsymbol{n}^{K} d \mathbf{s}=\int_{\partial K} \boldsymbol{\lambda}_{H} d \mathbf{s}=\int_{K} \boldsymbol{f} d \boldsymbol{x}
$$

where we used the first equation in (3.8).

In order to make the MHM method effective, one must provide approximate solutions for the local problems (3.2), (3.3) and (3.4). This is the subject of the next section.

3.3. The two-level MHM method. We begin selecting local finite dimensional spaces $\mathbf{V}_{h}(K) \subset \mathbf{V}_{0}^{\perp}(K)$ and $Q_{h}(K) \subset Q(K)$, given by

$$
\mathbf{V}_{h}(K):=\left\{\boldsymbol{v}_{h} \in \mathbf{V}_{0}^{\perp}(K) \cap C^{0}(K)^{d}:\left.\boldsymbol{v}_{h}\right|_{\tau} \in \mathbb{P}_{k}(\tau)^{d} \text { for all } \tau \in \mathcal{T}_{h}^{K}\right\}
$$

and

$$
Q_{h}(K):=\left\{q_{h} \in Q(K) \cap C^{0}(K):\left.q_{h}\right|_{\tau} \in \mathbb{P}_{n}(\tau) \text { for all } \tau \in \mathcal{T}_{h}^{K}\right\}
$$


where $\mathbb{P}_{r}(\tau)$ is the polynomial space of functions defined on $\tau \in \mathcal{T}_{h}^{K}$ with total degree less or equal to $r$. The corresponding global finite dimensional space are given by

$$
\mathbf{V}_{h}:=\oplus_{K \in \mathcal{T}_{H}} \mathbf{V}_{h}(K) \quad \text { and } \quad Q_{h}:=\oplus_{K \in \mathcal{T}_{H}} Q_{h}(K) .
$$

The two-level MHM method reads: Find $\left(\boldsymbol{u}_{0}^{H, h}, \boldsymbol{\lambda}_{H, h}, \rho_{H}\right) \in \mathbf{V}_{0} \times \boldsymbol{\Lambda}_{H} \times \mathbb{R}$ such that

$$
\left\{\begin{aligned}
\left(\boldsymbol{\lambda}_{H, h}, \boldsymbol{v}_{0}\right)_{\partial \mathcal{T}_{H}} & =\left(\boldsymbol{f}, \boldsymbol{v}_{0}\right)_{\mathcal{T}_{H}}, \\
\left(\boldsymbol{\mu}_{H}, \boldsymbol{u}_{0}^{H, h}\right)_{\partial \mathcal{T}_{H}}+\left(\boldsymbol{\mu}_{H}, T_{h}^{u} \boldsymbol{\lambda}_{H, h}\right)_{\partial \mathcal{T}_{H}}+\left(\boldsymbol{\mu}_{H}, \bar{T}_{h}^{u} \rho_{H}\right)_{\partial \mathcal{T}_{H}} & =\left\langle\boldsymbol{\mu}_{H}, \boldsymbol{g}\right\rangle_{\partial \Omega}-\left(\boldsymbol{\mu}_{H}, \hat{T}^{u} \boldsymbol{f}\right)_{\partial \mathcal{T}_{H}}, \\
\left(\xi_{H}, T_{h}^{p} \boldsymbol{\lambda}_{H, h}\right)_{\Omega}+\left(\xi_{H}, \bar{T}_{h}^{p} \rho_{H}\right)_{\Omega} & =-\left(\xi_{H}, \hat{T}_{h}^{p} \boldsymbol{f}\right)_{\Omega},
\end{aligned}\right.
$$

for all $\left(\boldsymbol{v}_{0}, \boldsymbol{\mu}_{H}, \xi_{H}\right) \in \mathbf{V}_{0} \times \boldsymbol{\Lambda}_{H} \times \mathbb{R}$.

Operators $\boldsymbol{T}_{h}, \overline{\boldsymbol{T}}_{h}$ and $\hat{\boldsymbol{T}}_{h}$ are defined such that they approximate $\boldsymbol{T}, \overline{\boldsymbol{T}}$ and $\hat{\boldsymbol{T}}$ when $h$ goes to zero. In this work, one adopts the unusual stabilized finite element method (USFEM) proposed in [10] to define $\boldsymbol{T}_{h}$, $\overline{\boldsymbol{T}}_{h}$ and $\hat{\boldsymbol{T}}_{h}$. The USFEM allows using nodal equal-order pair of spaces for the velocity and the pressure variables, i.e., we can set $k=n$ in (3.10) and (3.11). It is worth to mention that, in its more general version, the MHM method may adopt different order of interpolation spaces in each $K \in \mathcal{T}_{H}$ (and even different numerical methods). Here, for the sake of clarity, we adopt the USFEM method with the same degree of interpolation in all elements of the partition.

We recall (see [10] for details) that the USFEM consists of finding $(\boldsymbol{u}, p) \in \mathbf{V}_{h}(K) \times Q_{h}(K)$ such that

$$
\tilde{B}_{K}((\boldsymbol{u}, p),(\boldsymbol{v}, q))=F_{K}(\boldsymbol{v}, q) \text { for all }(\boldsymbol{v}, q) \in \mathbf{V}_{h}(K) \times Q_{h}(K),
$$

where

$$
\begin{aligned}
\tilde{B}_{K}((\boldsymbol{u}, p),(\boldsymbol{v}, q)):= & a_{K}(\boldsymbol{u}, \boldsymbol{v})+b_{K}(\boldsymbol{v}, p)-b_{K}(\boldsymbol{u}, q) \\
& -\sum_{\tau \in \mathcal{T}_{h}^{K}} \kappa_{\tau}(-\nu \Delta \boldsymbol{u}+\boldsymbol{\gamma} \boldsymbol{u}+\nabla p,-\nu \Delta \boldsymbol{v}+\boldsymbol{\gamma} \boldsymbol{v}-\nabla q)_{\tau}
\end{aligned}
$$

and

$$
F_{K}(\boldsymbol{v}, q):=(\boldsymbol{f}, \boldsymbol{v})_{K}-\sum_{\tau \in \mathcal{T}_{h}^{K}} \kappa_{\tau}(\boldsymbol{f},-\nu \Delta \boldsymbol{v}+\boldsymbol{\gamma} \boldsymbol{v}-\nabla q)_{\tau} .
$$

The stabilization parameter is given by

$$
\kappa_{\tau}:=\frac{h_{\tau}^{2}}{\gamma_{\min } h_{\tau}^{2} \max \left\{1, \lambda_{\tau}\right\}+\frac{4 \nu}{m_{k}}} \quad \text { with } \quad \lambda_{\tau}:=\frac{4 \nu}{m_{k} \gamma_{\min } h_{\tau}^{2}},
$$

where $\gamma_{\min }$ is defined in $(2.2), m_{k}:=\min \left\{\frac{1}{3}, C_{k}\right\}$ and

$$
C_{k} h_{\tau}^{2}\|\Delta \boldsymbol{v}\|_{0, \tau}^{2} \leq\|\nabla \boldsymbol{v}\|_{0, \tau}^{2} \quad \text { for all } \boldsymbol{v} \in \mathbf{V}_{h}(K) .
$$

Note that the only unknown constant in the design of the stabilization parameter is $C_{k}$. This constant, depending only on $d$ and the polynomial degree for the velocity, is tabulated in [23] for some 2D cases. A general way to calculate $C_{k}$ numerically is proposed in [20].

Owing to the above definitions, we define the corresponding discrete operators as follows:

- $\boldsymbol{T}_{h}: \boldsymbol{\Lambda}_{H} \rightarrow \mathbf{V}_{h} \times Q_{h}$ such that $\left.\boldsymbol{T}_{h} \boldsymbol{\lambda}_{H, h}\right|_{K}:=\left(T_{h}^{u} \boldsymbol{\lambda}_{H, h}, T_{h}^{p} \boldsymbol{\lambda}_{H, h}\right)$ solves

$$
\tilde{B}_{K}\left(\left(T_{h}^{u} \boldsymbol{\lambda}_{H, h}, T_{h}^{p} \boldsymbol{\lambda}_{H, h}\right),\left(\boldsymbol{v}_{h}, q_{h}\right)\right)=-\left\langle\boldsymbol{\lambda}_{H, h}, \boldsymbol{v}_{h}\right\rangle_{\partial K} \quad \text { for all }\left(\boldsymbol{v}_{h}, q_{h}\right) \in \mathbf{V}_{h}(K) \times Q_{h}(K) ;
$$

- $\hat{\boldsymbol{T}}_{h}: L^{2}(\Omega)^{d} \rightarrow \mathbf{V}_{h} \times Q_{h}$ such that $\left.\hat{\boldsymbol{T}}_{h} \boldsymbol{f}\right|_{K}:=\left(\hat{T}_{h}^{\boldsymbol{u}} \boldsymbol{f}, \hat{T}_{h}^{p} \boldsymbol{f}\right)$ solves

$$
\tilde{B}_{K}\left(\left(\hat{T}_{h}^{u} \boldsymbol{f}_{H}, \hat{T}_{h}^{p} \boldsymbol{f}\right),\left(\boldsymbol{v}_{h}, q_{h}\right)\right)=F_{K}\left(\boldsymbol{v}_{h}, q_{h}\right) \quad \text { for all }\left(\boldsymbol{v}_{h}, q_{h}\right) \in \mathbf{V}_{h}(K) \times Q_{h}(K) ;
$$

- $\overline{\boldsymbol{T}}_{h}: \mathbb{R} \rightarrow \mathbf{V}_{h} \times Q_{h}$ such that $\left.\overline{\boldsymbol{T}}_{h} \rho_{H}\right|_{K}:=\left(\bar{T}_{h}^{u} \rho_{H}, \bar{T}_{h}^{p} \rho_{H}\right)$ solves

$$
\tilde{B}_{K}\left(\left(\bar{T}_{h}^{u} \rho_{H}, \bar{T}_{h}^{p} \rho_{H}\right),\left(\boldsymbol{v}_{h}, q_{h}\right)\right)=-\left(\rho_{H}, q_{h}\right)_{K} \quad \text { for all }\left(\boldsymbol{v}_{h}, q_{h}\right) \in \mathbf{V}_{h}(K) \times Q_{h}(K) \text {. }
$$


Operators $\boldsymbol{T}_{h}, \hat{\boldsymbol{T}}_{h}$ and $\overline{\boldsymbol{T}}_{h}$ are well-defined since the discrete problems (3.17), (3.18) and (3.19) are wellposed from [10]. As a result, the discrete two-level solution $\left(\boldsymbol{u}_{H, h}, p_{H, h}, \rho_{H}\right)$ is given through the expressions

$$
\boldsymbol{u}_{H, h}:=\boldsymbol{u}_{0}^{H, h}+T_{h}^{\boldsymbol{u}} \boldsymbol{\lambda}_{H, h}+\hat{T}_{h}^{u} \boldsymbol{f} \quad \text { and } \quad p_{H, h}:=T_{h}^{p} \boldsymbol{\lambda}_{H, h}+\hat{T}_{h}^{p} \boldsymbol{f},
$$

where we used that $\rho_{H}=0$ (c.f. [7]).

Remark 2. By taking $\left(\boldsymbol{v}_{h}, q_{h}\right)=\left(0,1_{K}\right)$ in equations (3.17), (3.18) and (3.19), the following local conservation property holds for the discrete velocity field $\boldsymbol{u}_{H, h}$

$$
\int_{K} \nabla \cdot \boldsymbol{u}_{H, h} d \boldsymbol{x}=0 \quad \text { for all } K \in \mathcal{T}_{H},
$$

and the approximate flux $\boldsymbol{\lambda}_{H, h}$ is in equilibrium with external forces, i.e.,

$$
\int_{\partial K} \boldsymbol{\lambda}_{H, h} d \mathbf{s}=\int_{K} \boldsymbol{f} d \boldsymbol{x} \quad \text { for all } K \in \mathcal{T}_{H},
$$

\section{A POSTERIORI ERROR ANALYSIS}

This section introduces the multiscale a posteriori error estimator, which is proved to be locally efficient and reliable.

4.1. The multiscale a posteriori error estimator. This section presents the residual-based error estimator for the MHM method. Given $F \in \mathcal{E}_{H}$, we define a face-residual estimator as follows:

$$
\boldsymbol{R}_{F}:= \begin{cases}-\frac{1}{2} \llbracket \boldsymbol{u}_{H, h} \rrbracket & F \in \mathcal{E}_{0}, \\ \boldsymbol{g}-\boldsymbol{u}_{H, h} & F \in \mathcal{E}_{D},\end{cases}
$$

where the jump over a face $F \in \mathcal{E}_{0}$ of a vector $\boldsymbol{v} \in \mathbf{V}$ is defined by

$$
\llbracket \boldsymbol{v} \rrbracket:=\left.\left(\left.\boldsymbol{v}\right|_{K^{+}}\right)\right|_{F}-\left.\left(\left.\boldsymbol{v}\right|_{K^{-}}\right)\right|_{F}
$$

with $K^{+}$and $K^{-}$are the two elements sharing $F$. Thus, we define

$$
\eta_{1, F}:=\left\{\sum_{\tilde{F} \in \mathcal{T}_{\tilde{H}}(F)} \eta_{1, \tilde{F}}^{2}\right\}^{1 / 2}
$$

where

$$
\eta_{1, \tilde{F}}:=\frac{\left\|\boldsymbol{R}_{F}\right\|_{0, \tilde{F}}}{H_{F}^{1 / 2}}
$$

for all $\tilde{F} \in \mathcal{T}_{\tilde{H}}(F)$. Owing to previous definitions, the first level a posteriori error estimator reads

$$
\eta_{1}:=\left\{\sum_{K \in \mathcal{T}_{H}} \eta_{1, K}^{2}\right\}^{1 / 2}, \quad \text { where } \quad \eta_{1, K}^{2}:=\sum_{F \subset \partial K} \eta_{1, F}^{2} .
$$

To propose a second-level estimator, we first need some notations related to local residuals in each subelement $\tau \in \mathcal{T}_{h}^{K}$ and on each sub-face $\zeta \in \mathcal{E}_{h}^{K}$. They are the following:

$$
\boldsymbol{R}_{\tau}^{K}:=\left.\left(\nu \Delta \boldsymbol{u}_{H, h}-\boldsymbol{\gamma} \boldsymbol{u}_{H, h}-\nabla p_{H, h}+\boldsymbol{f}\right)\right|_{\tau} \quad \text { for all } \tau \in \mathcal{T}_{h}^{K}
$$

and

$$
\boldsymbol{R}_{\zeta}^{K}:= \begin{cases}\llbracket-\nu \frac{\partial \boldsymbol{u}_{H, h}}{\partial \boldsymbol{n}_{\zeta}^{\tau}}+p_{H, h} \boldsymbol{n}_{\zeta}^{\tau} \rrbracket & \text { on } \zeta \in \mathcal{E}_{0}^{K} \\ -\boldsymbol{\lambda}_{H, h}-\nu \frac{\partial \boldsymbol{u}_{H, h}}{\partial \boldsymbol{n}_{\zeta}^{\tau}}+p_{H, h} \boldsymbol{n}_{\zeta}^{\tau} & \text { on } \zeta \in \mathcal{E}_{h}^{K} \backslash \mathcal{E}_{0}^{K} .\end{cases}
$$

Using previous definitions, the second-level a posteriori error estimator reads as follows

$$
\eta_{2}:=\left\{\sum_{K \in \mathcal{T}_{H}} \eta_{2, K}^{2}\right\}^{1 / 2}
$$


where

$$
\eta_{2, K}:=\left[\sum_{\tau \in \mathcal{T}_{h}^{K}}\left[h_{\tau}^{2}\left\|\boldsymbol{R}_{\tau}^{K}\right\|_{0, \tau}^{2}+\left\|\nabla \cdot \boldsymbol{u}_{H, h}\right\|_{0, \tau}^{2}\right]+\sum_{\zeta \in \mathcal{E}_{h}^{K}} h_{\zeta}\left\|\boldsymbol{R}_{\zeta}^{K}\right\|_{0, \zeta}^{2}\right]^{1 / 2} .
$$

Summing up (4.3) and (4.6), we build an a posteriori error estimator, which includes the first and second level residuals, given by

$$
\eta=\eta_{1}+\eta_{2} .
$$

Remark 3. The contribution of $\eta_{1}$ depends only on the jump of the velocity across the faces of the first-level mesh. It has the same form of the a posteriori estimator introduced in [6] for the Darcy equation. We recall that the error associated to the local problems were not considered in [6], which explains the absence of contribution $\eta_{2}$ in [6].

Remark 4. Note that $\eta_{2}$ behaves asymptotically as a high order term compare to $\eta$ when $h \leq H^{\alpha+1}$, with $\alpha>0$. With such a choice, second-level error estimator $\eta_{2}$ may be disregarded in the numerical tests, i.e., $\eta=\eta_{1}$. As shown in the next section, such a simplified version is also an efficient and reliable a posteriori error estimator for the two-level MHM method.

4.2. Efficiency and reliability. This section proves that the estimator (4.7) is close to the real error. To this end, we need some intermediary estimates which are addressed first.

The first result mesures the quality of the second-level approximation with respect to the second-level a posteriori error estimator $\eta_{2, K}$ in each $K \in \mathcal{T}_{H}$.

Lemma 4.1. The following estimate holds

$$
\left\|\left(\left(T^{\boldsymbol{u}}-T_{h}^{\boldsymbol{u}}\right) \boldsymbol{\lambda}_{H, h}+\left(\hat{T}^{\boldsymbol{u}}-\hat{T}_{h}^{\boldsymbol{u}}\right) \boldsymbol{f},\left(T^{p}-T_{h}^{p}\right) \boldsymbol{\lambda}_{H, h}+\left(\hat{T}^{p}-\hat{T}_{h}^{p}\right) \boldsymbol{f}\right)\right\|_{\mathbf{V}(K) \times Q(K)} \preceq \eta_{2, K} .
$$

Proof. Recalling from (3.20) that

$$
p_{H, h}=T_{h}^{p} \boldsymbol{\lambda}_{H, h}+\hat{T}_{h}^{p} \boldsymbol{f}
$$

we define

$$
\left\{\begin{aligned}
\tilde{\boldsymbol{u}} & :=T^{\boldsymbol{u}} \boldsymbol{\lambda}_{H, h}+\hat{T}^{u} \boldsymbol{f} \\
\tilde{p} & :=T^{p} \boldsymbol{\lambda}_{H, h}+\hat{T}^{p} \boldsymbol{f} \\
\tilde{\boldsymbol{u}}_{H, h} & :=T_{h}^{\boldsymbol{u}} \boldsymbol{\lambda}_{H, h}+\hat{T}_{h}^{u} \boldsymbol{f}
\end{aligned}\right.
$$

and

$$
\left\{\begin{array}{l}
e^{\tilde{\boldsymbol{u}}}:=\tilde{\boldsymbol{u}}-\tilde{\boldsymbol{u}}_{H, h}, \\
e^{\tilde{p}}:=\tilde{p}-p_{H, h} .
\end{array}\right.
$$

Let $\boldsymbol{v} \in \mathbf{V}(K), q \in Q(K)$ and $\boldsymbol{v}_{h}:=\mathscr{C}_{h}^{K}(\boldsymbol{v})$ be the Clément interpolant of $\boldsymbol{v}$ on $K \in \mathcal{T}_{H}$. From the definition of the bilinear form $B_{K}(\cdot, \cdot)$ given in (3.1), (3.2), (3.3), (3.20) and integration by parts, it holds

$$
\begin{aligned}
B_{K}\left(\left(e^{\tilde{\boldsymbol{u}}}, e^{\tilde{p}}\right),(\boldsymbol{v}, q)\right) & =a_{K}\left(\tilde{\boldsymbol{u}}-\tilde{\boldsymbol{u}}_{H, h}, \boldsymbol{v}\right)+b_{K}\left(\boldsymbol{v}, \tilde{p}-p_{H, h}\right)-b_{K}\left(\tilde{\boldsymbol{u}}-\tilde{\boldsymbol{u}}_{H, h}, q\right) \\
& =-\left\langle\boldsymbol{\lambda}_{H, h}, \boldsymbol{v}\right\rangle_{\partial K}+(\boldsymbol{f}, \boldsymbol{v})_{K}-\left[a_{K}\left(\boldsymbol{u}_{H, h}, \boldsymbol{v}\right)+b_{K}\left(\boldsymbol{v}, p_{H, h}\right)-b_{K}\left(\boldsymbol{u}_{H, h}, q\right)\right] \\
& =\sum_{\tau \in \mathcal{T}_{h}^{K}}\left[\left(\boldsymbol{R}_{\tau}^{K}, \boldsymbol{v}\right)_{\tau}+\left(\nabla \cdot \boldsymbol{u}_{H, h}, q\right)_{\tau}\right]+\sum_{\zeta \in \mathcal{E}_{h}^{K}}\left(\boldsymbol{R}_{\zeta}^{K}, \boldsymbol{v}\right)_{\zeta},
\end{aligned}
$$

thus, replacing $\boldsymbol{v}$ by $\boldsymbol{v}-\boldsymbol{v}_{h}$ in (4.8) and using Cauchy-Schwartz inequality, we get

$$
\begin{aligned}
B_{K}\left(\left(e^{\tilde{\boldsymbol{u}}}, e^{\tilde{p}}\right),\left(\boldsymbol{v}-\boldsymbol{v}_{h}, q\right)\right) \leq & \sum_{\tau \in \mathcal{T}_{h}^{K}}\left[\left\|\boldsymbol{R}_{\tau}^{K}\right\|_{0, \tau}\left\|\boldsymbol{v}-\boldsymbol{v}_{h}\right\|_{0, \tau}+\left\|\nabla \cdot \boldsymbol{u}_{H, h}\right\|_{0, \tau}\|q\|_{0, \tau}\right] \\
& +\sum_{\zeta \in \mathcal{E}_{h}^{K}}\left\|\boldsymbol{R}_{\zeta}^{K}\right\|_{0, \zeta}\left\|\boldsymbol{v}-\boldsymbol{v}_{h}\right\|_{0, \zeta} .
\end{aligned}
$$


On the other hand, testing (4.8) with $(\boldsymbol{v}, q)=\left(\boldsymbol{v}_{h}, 0\right) \in \mathbf{V}_{h} \times Q_{h}$, we arrive at

$$
\begin{aligned}
B_{K}\left(\left(e^{\tilde{\boldsymbol{u}}}, e^{\tilde{p}}\right),\left(\boldsymbol{v}_{h}, 0\right)\right) & =a_{K}\left(\tilde{\boldsymbol{u}}-\tilde{\boldsymbol{u}}_{H, h}, \boldsymbol{v}_{h}\right)+b_{K}\left(\boldsymbol{v}_{h}, \tilde{p}-p_{H, h}\right) \\
& =-\sum_{\tau \in \mathcal{T}_{h}^{K}} \kappa_{\tau}\left(-\nu \Delta \boldsymbol{u}_{H, h}+\boldsymbol{\gamma} \boldsymbol{u}_{H, h}+\nabla p_{H, h}-\boldsymbol{f},-\nu \Delta \boldsymbol{v}_{h}+\gamma \boldsymbol{v}_{h}\right)_{\tau} \\
& =\sum_{\tau \in \mathcal{T}_{h}^{K}} \kappa_{\tau}\left(\boldsymbol{R}_{\tau}^{K},-\nu \Delta \boldsymbol{v}_{h}+\boldsymbol{\gamma} \boldsymbol{v}_{h}\right)_{\tau} .
\end{aligned}
$$

From the definition of $\kappa_{\tau}$ (see (3.15)), it is easy to verify that

$$
\kappa_{\tau} \leq \frac{h_{\tau}^{2}}{12 \nu}
$$

thus, using (3.16) and (4.11), we get

$$
\begin{aligned}
\kappa_{\tau}\left\|-\nu \Delta \boldsymbol{v}_{h}+\boldsymbol{\gamma} \boldsymbol{v}_{h}\right\|_{0, \tau} & \leq \frac{h_{\tau}}{C_{k} 12 \nu}\left\|\nabla \boldsymbol{v}_{h}\right\|_{0, \tau}+\|\boldsymbol{\gamma}\|_{\infty} \frac{h_{\tau}^{2}}{12 \nu}\left\|\boldsymbol{v}_{h}\right\|_{0, \tau} \\
& \preceq h_{\tau}\left\{\left\|\nabla \boldsymbol{v}_{h}\right\|_{0, \tau}+\frac{1}{d_{\Omega}}\left\|\boldsymbol{v}_{h}\right\|_{0, \tau}\right\} .
\end{aligned}
$$

Then, combining (4.9), (4.10), (4.12), the properties of Clément interpolation operator (2.23)-(2.25) and the mesh regularity, we arrive at

$$
\begin{aligned}
B_{K}\left(\left(e^{\tilde{\boldsymbol{u}}}, e^{\tilde{p}}\right),(\boldsymbol{v}, q)\right)= & B_{K}\left(\left(e^{\tilde{\boldsymbol{u}}}, e^{\tilde{p}}\right),\left(\boldsymbol{v}-\boldsymbol{v}_{h}, q\right)\right)+B_{K}\left(\left(e^{\tilde{\boldsymbol{u}}}, e^{\tilde{p}}\right),\left(\boldsymbol{v}_{h}, 0\right)\right) \\
\preceq & \sum_{\tau \in \mathcal{T}_{h}^{K}}\left[\left\|\boldsymbol{R}_{\tau}^{K}\right\|_{0, \tau}\left\|\boldsymbol{v}-\boldsymbol{v}_{h}\right\|_{0, \tau}+\left\|\nabla \cdot \boldsymbol{u}_{H, h}\right\|_{0, \tau}\|q\|_{0, \tau}\right] \\
& +\sum_{\zeta \in \mathcal{E}_{h}^{K}}\left\|\boldsymbol{R}_{\zeta}^{K}\right\|_{0, \zeta}\left\|\boldsymbol{v}-\boldsymbol{v}_{h}\right\|_{0, \zeta}+\sum_{\tau \in \mathcal{T}_{h}^{K}} h_{\tau}\left\|\boldsymbol{R}_{\tau}^{K}\right\|_{0, \tau}\left\{\left\|\nabla \boldsymbol{v}_{h}\right\|_{0, \tau}+\frac{1}{d_{\Omega}}\left\|\boldsymbol{v}_{h}\right\|_{0, \tau}\right\} \\
\preceq & \sum_{\tau \in \mathcal{T}_{h}^{K}}\left[h_{\tau}\left\|\boldsymbol{R}_{\tau}^{K}\right\|_{0, \tau}|\boldsymbol{v}|_{1, \tilde{\omega}_{\tau}}+\left\|\nabla \cdot \boldsymbol{u}_{H, h}\right\|_{0, \tau}\|q\|_{0, \tau}\right] \\
& +\sum_{\zeta \in \mathcal{E}_{h}^{K}} h_{\zeta}^{1 / 2}\left\|\boldsymbol{R}_{\zeta}^{K}\right\|_{0, \zeta}|\boldsymbol{v}|_{1, \tilde{\omega}_{\zeta}}+\sum_{\tau \in \mathcal{T}_{h}^{K}} h_{\tau}\left\|\boldsymbol{R}_{\tau}^{K}\right\|_{0, \tau}\left\{\left\|\nabla \boldsymbol{v}_{h}\right\|_{0, \tau}+\frac{1}{d_{\Omega}}\left\|\boldsymbol{v}_{h}\right\|_{0, \tau}\right\} \\
\preceq & {\left[\sum_{\tau \in \mathcal{T}_{h}^{K}}\left[h_{\tau}^{2}\left\|\boldsymbol{R}_{\tau}^{K}\right\|_{0, \tau}^{2}+\left\|\nabla \cdot \boldsymbol{u}_{H, h}\right\|_{0, \tau}^{2}\right]+\sum_{\zeta \in \mathcal{E}_{h}^{K}} h_{\zeta}\left\|\boldsymbol{R}_{\zeta}^{K}\right\|_{0, \zeta}^{2}\right]^{1 / 2} } \\
& \times\left[\sum_{\tau \in \mathcal{T}_{h}^{K}}\left[\|\nabla \boldsymbol{v}\|_{0, \tau}^{2}+\frac{1}{d_{\Omega}^{2}}\left\|\boldsymbol{v}_{h}\right\|_{0, \tau}^{2}+|\boldsymbol{v}|_{1, \tilde{\omega}_{\tau}}^{2}+\|q\|_{0, \tau}^{2}\right]+\sum_{\zeta \in \mathcal{E}_{h}^{K}}|\boldsymbol{v}|_{1, \tilde{\omega}_{\zeta}}^{2}\right]^{1 / 2} \\
\preceq & {\left[\sum_{\tau \in \mathcal{T}_{h}^{K}}\left[h_{\tau}^{2}\left\|\boldsymbol{R}_{\tau}^{K}\right\|_{0, \tau}^{2}+\left\|\nabla \cdot \boldsymbol{u}_{H, h}\right\|_{0, \tau}^{2}\right]+\sum_{\zeta \in \mathcal{E}_{h}^{K}} h_{\zeta}\left\|\boldsymbol{R}_{\zeta}^{K}\right\|_{0, \zeta}^{2}\right]^{1 / 2} \times\|(\boldsymbol{v}, q)\|_{\mathbf{v}(K) \times Q(K)} . }
\end{aligned}
$$

Thus, the result follows from the inf-sup condition given in Theorem 2.2.

Now, we adapt the ideas of the proof in [6, Lemma 5.1] to include second-level approximations.

Lemma 4.2. Let $\boldsymbol{u}_{H, h} \in \mathbf{V}_{h}$ be the discrete solution given by (3.20). Then, there exists $\boldsymbol{\chi} \in \mathbf{V}$ satisfying

$$
(\boldsymbol{\mu}, \boldsymbol{\chi})_{\partial \mathcal{T}_{H}}=\langle\boldsymbol{\mu}, \boldsymbol{g}\rangle_{\partial \Omega}-\left(\boldsymbol{\mu}, \boldsymbol{u}_{H, h}\right)_{\partial \mathcal{T}_{H}} \quad \text { for all } \boldsymbol{\mu} \in \boldsymbol{\Lambda},
$$

such that

$$
\|\chi\|_{\mathrm{V}} \preceq \eta_{1} .
$$

Proof. Let $(\overline{\boldsymbol{\chi}}, \overline{\boldsymbol{\xi}}) \in \mathbf{V} \times \mathbf{\Lambda}$ be the solution of the following problem

$$
\begin{aligned}
\frac{1}{d_{\Omega}^{2}}(\overline{\boldsymbol{\chi}}, \boldsymbol{v})_{\mathcal{T}_{H}}+(\nabla \overline{\boldsymbol{\chi}}, \nabla \boldsymbol{v})_{\mathcal{T}_{H}}+(\overline{\boldsymbol{\xi}}, \boldsymbol{v})_{\partial \mathcal{T}_{H}} & =\frac{1}{d_{\Omega}^{2}}\left(\boldsymbol{u}_{H, h}, \boldsymbol{v}\right)_{\mathcal{T}_{H}}-\sum_{K \in \mathcal{T}_{H}} \sum_{\tau \in \mathcal{T}_{h}^{K}}\left(\Delta \boldsymbol{u}_{H, h}, \boldsymbol{v}\right)_{\tau} \\
(\boldsymbol{\mu}, \overline{\boldsymbol{\chi}})_{\partial \mathcal{T}_{H}} & =\langle\boldsymbol{\mu}, \boldsymbol{g}\rangle_{\partial \Omega}
\end{aligned}
$$


for all $(\boldsymbol{v}, \boldsymbol{\mu}) \in \mathbf{V} \times \boldsymbol{\Lambda}$. From [22] we have that $\overline{\boldsymbol{\xi}}$ belongs to $L^{2}\left(\mathcal{E}_{H}\right)^{d}$. Then, there exists $\boldsymbol{\sigma} \in H(\operatorname{div} ; \Omega)$ such that $\boldsymbol{\sigma} \boldsymbol{n}=\overline{\boldsymbol{\xi}}$ on $\mathcal{E}_{H}$, and we define $\tilde{\boldsymbol{\xi}}:=\boldsymbol{\sigma} \boldsymbol{n}_{\zeta}^{\tau}$ on $\mathcal{E}_{h}^{K}$. Using $\left.\boldsymbol{v}\right|_{K} \in H^{1}(K)^{d}$, we can rewrite (4.13) as follows

$$
\begin{aligned}
\frac{1}{d_{\Omega}^{2}}(\bar{\chi}, \boldsymbol{v})_{\mathcal{T}_{H}}+(\nabla \overline{\boldsymbol{\chi}}, \nabla \boldsymbol{v})_{\mathcal{T}_{H}}+\sum_{K \in \mathcal{T}_{H}} \sum_{\tau \in \mathcal{T}_{h}^{K}}\langle\tilde{\boldsymbol{\xi}}, \boldsymbol{v}\rangle_{\partial \tau} & =\frac{1}{d_{\Omega}^{2}}\left(\boldsymbol{u}_{H, h}, \boldsymbol{v}\right)_{\mathcal{T}_{H}}-\sum_{K \in \mathcal{T}_{H}} \sum_{\tau \in \mathcal{T}_{h}^{K}}\left(\Delta \boldsymbol{u}_{H, h}, \boldsymbol{v}\right)_{\tau} \\
(\boldsymbol{\mu}, \overline{\boldsymbol{\chi}})_{\partial \mathcal{T}_{H}} & =\langle\boldsymbol{\mu}, \boldsymbol{g}\rangle_{\partial \Omega}
\end{aligned}
$$

for all $(\boldsymbol{v}, \boldsymbol{\mu}) \in \mathbf{V} \times \boldsymbol{\Lambda}$. Now, define $\boldsymbol{\chi}:=\overline{\boldsymbol{\chi}}-\boldsymbol{u}_{H, h}$ and $\boldsymbol{\xi}:=\tilde{\boldsymbol{\xi}}+\nabla \boldsymbol{u}_{H, h} \boldsymbol{n}$. Then, $(\boldsymbol{\chi}, \boldsymbol{\xi}) \in H^{3 / 2}\left(\mathcal{T}_{H}\right)^{d} \times L^{2}\left(\mathcal{E}_{H}\right)^{d}$ and satisfies

$$
\begin{aligned}
\frac{1}{d_{\Omega}^{2}}(\boldsymbol{\chi}, \boldsymbol{v})_{\mathcal{T}_{H}}+(\nabla \boldsymbol{\chi}, \nabla \boldsymbol{v})_{\mathcal{T}_{H}}+(\boldsymbol{\xi}, \boldsymbol{v})_{\partial \mathcal{T}_{H}}=0 & \text { for all } \boldsymbol{v} \in \mathbf{V} \\
(\boldsymbol{\mu}, \boldsymbol{\chi})_{\partial \mathcal{T}_{H}}=-\left(\boldsymbol{\mu}, \boldsymbol{u}_{H, h}\right)_{\partial \mathcal{T}_{H}}+\langle\boldsymbol{\mu}, \boldsymbol{g}\rangle_{\partial \Omega} & \text { for all } \boldsymbol{\mu} \in \boldsymbol{\Lambda} .
\end{aligned}
$$

Testing (4.14) with $(\boldsymbol{v}, \boldsymbol{\mu})=(\boldsymbol{\chi}, \boldsymbol{\xi})$ and using Cauchy-Schwartz inequality, we get

$$
\begin{aligned}
\|\boldsymbol{\chi}\|_{\mathbf{v}}^{2} & =\sum_{K \in \mathcal{T}_{H}}\left\{\frac{1}{d_{\Omega}^{2}}\|\boldsymbol{\chi}\|_{0, K}^{2}+\|\nabla \boldsymbol{\chi}\|_{0, K}^{2}\right\}=-(\boldsymbol{\xi}, \boldsymbol{\chi})_{\partial \mathcal{T}_{H}}=\left(\boldsymbol{\xi}, \boldsymbol{u}_{H, h}\right)_{\partial \mathcal{T}_{H}}-(\boldsymbol{\xi}, \boldsymbol{g})_{\partial \Omega} \\
& =\sum_{F \in \mathcal{E}_{0}}\|\boldsymbol{\xi}\|_{0, F}\left\|\llbracket \boldsymbol{u}_{H, h} \rrbracket\right\|_{0, F}+\sum_{F \in \partial \Omega}\|\boldsymbol{\xi}\|_{0, F}\left\|\boldsymbol{g}-\boldsymbol{u}_{H, h}\right\|_{0, F} \\
& \leq\left\{\sum_{F \in \mathcal{E}_{H}} H_{F}\|\boldsymbol{\xi}\|_{0, F}^{2}\right\}^{1 / 2}\left\{\sum_{F \in \mathcal{E}_{0}} \frac{1}{H_{F}}\left\|\llbracket \boldsymbol{u}_{H, h} \rrbracket\right\|_{0, F}^{2}+\sum_{F \in \partial \Omega} \frac{1}{H_{F}}\left\|\boldsymbol{g}-\boldsymbol{u}_{H, h}\right\|_{0, F}^{2}\right\}^{1 / 2} \\
& =\left\{\sum_{F \in \mathcal{E}_{H}} H_{F}\|\nabla \boldsymbol{\chi} \boldsymbol{n}\|_{0, F}^{2}\right\}^{1 / 2} \eta_{1} \preceq\|\boldsymbol{\chi}\|_{\mathbf{v}} \eta_{1}
\end{aligned}
$$

and the result follows.

In what follows, we will use the following local norm

$$
\|\boldsymbol{v}\|_{\mathbf{V}, \omega_{F}}^{2}:=\sum_{K \in \omega_{F}}\left(H_{K}^{-2}\|\boldsymbol{v}\|_{0, K}^{2}+\|\nabla \boldsymbol{v}\|_{0, K}^{2}\right)
$$

where $F \in \mathcal{E}_{H}$ and $\omega_{F}$ stands for the set of elements sharing the face $F$. The next theorem proves a bound for $\eta_{2, K}$ with respect to the local error.

Theorem 4.3. Let $K \in \mathcal{T}_{H}$. For $\tau \in \mathcal{T}_{h}^{K}$, then it holds

$$
h_{\tau}\left\|\boldsymbol{R}_{\tau}^{K}\right\|_{0, \tau} \preceq\left[h_{\tau}\left\|\boldsymbol{u}-\boldsymbol{u}_{H, h}\right\|_{0, \tau}+\left|\boldsymbol{u}-\boldsymbol{u}_{H, h}\right|_{1, \tau}+\left\|p-p_{H, h}\right\|_{0, \tau}\right]
$$

and

$$
\left\|\nabla \cdot \boldsymbol{u}_{H, h}\right\|_{0, \tau} \preceq\left|\boldsymbol{u}-\boldsymbol{u}_{H, h}\right|_{1, \tau} .
$$

Furthermore, for $\zeta \in \mathcal{E}_{0}^{K}$ we have that

$$
h_{\zeta}^{1 / 2}\left\|\boldsymbol{R}_{\zeta}^{K}\right\|_{0, \zeta} \preceq \sum_{\tau \in \omega_{\zeta}}\left\{\left|\boldsymbol{u}-\boldsymbol{u}_{H, h}\right|_{1, \tau}+h_{\tau}\left\|\boldsymbol{u}-\boldsymbol{u}_{H, h}\right\|_{0, \tau}+\left\|p-p_{H, h}\right\|_{0, \tau}\right\}
$$

and, for $\zeta \in \mathcal{E}_{h}^{K} \backslash \mathcal{E}_{0}^{K}$ we get

$$
h_{\zeta}^{1 / 2}\left\|\boldsymbol{R}_{\zeta}^{K}\right\|_{0, \zeta} \preceq \sum_{\tau \in \omega_{\zeta}}\left\{\left|\boldsymbol{u}-\boldsymbol{u}_{H, h}\right|_{1, \tau}+h_{\tau}\left\|\boldsymbol{u}-\boldsymbol{u}_{H, h}\right\|_{0, \tau}+\left\|p-p_{H, h}\right\|_{0, \tau}\right\}+\left\|\boldsymbol{\lambda}-\boldsymbol{\lambda}_{H, h}\right\|_{-1 / 2, \partial K} .
$$

Therefore, it holds

$$
\eta_{2, K}^{2} \preceq\left\|\boldsymbol{u}-\boldsymbol{u}_{H, h}\right\|_{\mathbf{V}(K)}^{2}+\left\|p-p_{H, h}\right\|_{Q(K)}^{2}+\left\|\boldsymbol{\lambda}-\boldsymbol{\lambda}_{H, h}\right\|_{-1 / 2, \partial K}^{2},
$$

for all $K \in \mathcal{T}_{H}$. 
Proof. Let $K \in \mathcal{T}_{H}, \tau \in \mathcal{T}_{h}^{K}$ and $\zeta \in \mathcal{E}_{h}^{K}$. We define $\boldsymbol{b}_{\tau}^{K}:=b_{\tau}^{K} \boldsymbol{R}_{\tau}^{K}$ and $\boldsymbol{b}_{\zeta}^{K}:=b_{\zeta}^{K} \boldsymbol{P}_{\zeta}^{K}\left(\boldsymbol{R}_{\zeta}^{K}\right)$, where $\boldsymbol{P}_{\zeta}^{K}: \mathbb{P}_{k}(\zeta)^{d} \rightarrow \mathbb{P}_{k}\left(\omega_{\zeta}\right)^{d}$ is a extension of functions defined on a face $\zeta$ to the patch $\omega_{\zeta}$, as in [5, Section 4.1]. Since $\left.\boldsymbol{b}_{\tau}^{K}\right|_{\tau} \in H_{0}^{1}(\tau)^{d}$, using integration by parts, (2.13) and Theorem 2.3 we obtain

$$
\begin{aligned}
\left(\boldsymbol{R}_{\tau}^{K}, \boldsymbol{b}_{\tau}^{K}\right)_{\tau} & =\left(\nu \Delta \boldsymbol{u}_{H, h}-\gamma \boldsymbol{u}_{H, h}-\nabla p_{H, h}+\boldsymbol{f}, \boldsymbol{b}_{\tau}^{K}\right)_{\tau} \\
& =\left(\nu \nabla\left(\boldsymbol{u}-\boldsymbol{u}_{H, h}\right), \nabla \boldsymbol{b}_{\tau}^{K}\right)_{\tau}+\left(\gamma\left(\boldsymbol{u}-\boldsymbol{u}_{H, h}\right), \boldsymbol{b}_{\tau}^{K}\right)_{\tau}-\left(\nabla \cdot \boldsymbol{b}_{\tau}^{K}, p-p_{H, h}\right)_{\tau} \\
& \preceq\left[h_{\tau}^{-1}\left|\boldsymbol{u}-\boldsymbol{u}_{H, h}\right|_{1, \tau}+\left\|\boldsymbol{u}-\boldsymbol{u}_{H, h}\right\|_{0, \tau}+h_{\tau}^{-1}\left\|p-p_{H, h}\right\|_{0, \tau}\right]\left\|\boldsymbol{R}_{\tau}^{K}\right\|_{0, \tau}
\end{aligned}
$$

and then

$$
h_{\tau}\left\|\boldsymbol{R}_{\tau}^{K}\right\|_{0, \tau} \preceq h_{\tau}\left\|\boldsymbol{u}-\boldsymbol{u}_{H, h}\right\|_{0, \tau}+\left|\boldsymbol{u}-\boldsymbol{u}_{H, h}\right|_{1, \tau}+\left\|p-p_{H, h}\right\|_{0, \tau} .
$$

Using Theorem 2.3 again, we arrive at

$$
\begin{aligned}
\left\|\nabla \cdot \boldsymbol{u}_{H, h}\right\|_{0, \tau}^{2} & \preceq\left(\nabla \cdot \boldsymbol{u}_{H, h}, b_{\tau}^{K} \nabla \cdot \boldsymbol{u}_{H, h}\right)_{\tau}=\left(\nabla \cdot\left(\boldsymbol{u}_{H, h}-\boldsymbol{u}\right), b_{\tau}^{K} \nabla \cdot \boldsymbol{u}_{H, h}\right)_{\Omega} \\
& =\left(\nabla \cdot\left(\boldsymbol{u}_{H, h}-\boldsymbol{u}\right), b_{\tau}^{K} \nabla \cdot \boldsymbol{u}_{H, h}\right)_{\tau} \\
& \preceq\left|\boldsymbol{u}_{H, h}-\boldsymbol{u}\right|_{1, \tau}\left\|\nabla \cdot \boldsymbol{u}_{H, h}\right\|_{0, \tau}
\end{aligned}
$$

and hence

$$
\left\|\nabla \cdot \boldsymbol{u}_{H, h}\right\|_{0, \tau} \preceq\left|\boldsymbol{u}-\boldsymbol{u}_{H, h}\right|_{1, \tau} .
$$

Now we proceed to bound the term $h_{\zeta}^{1 / 2}\left\|\boldsymbol{R}_{\zeta}^{K}\right\|_{0, \zeta}$. For $\zeta \in \mathcal{E}_{0}^{K}$, from (2.13) and using Theorem 2.4, we get

$$
\begin{aligned}
\left(\boldsymbol{R}_{\zeta}^{K}, \boldsymbol{b}_{\zeta}^{K}\right)_{\zeta}= & \sum_{\tau \in \omega_{\zeta}}\left\{-\left(\boldsymbol{R}_{\tau}^{K}, \boldsymbol{b}_{\zeta}^{K}\right)_{\tau}+\left(\nu \nabla\left(\boldsymbol{u}-\boldsymbol{u}_{H, h}\right), \nabla \boldsymbol{b}_{\zeta}^{K}\right)_{\tau}+\left(\gamma\left(\boldsymbol{u}-\boldsymbol{u}_{H, h}\right), \boldsymbol{b}_{\zeta}^{K}\right)_{\tau}\right. \\
& \left.-\left(\nabla \cdot \boldsymbol{b}_{\zeta}^{K}, p-p_{H, h}\right)_{\tau}\right\} \\
\preceq & \sum_{\tau \in \omega_{\zeta}}\left\{\left\|\boldsymbol{R}_{\tau}^{K}\right\|_{0, \tau}\left\|\boldsymbol{b}_{\zeta}^{K}\right\|_{0, \tau}+\nu\left|\boldsymbol{u}-\boldsymbol{u}_{H, h}\right|_{1, \tau}\left|\boldsymbol{b}_{\zeta}^{K}\right|_{1, \tau}+\left\|\gamma\left(\boldsymbol{u}-\boldsymbol{u}_{H, h}\right)\right\|_{0, \tau}\left\|\boldsymbol{b}_{\zeta}^{K}\right\|_{0, \tau}\right. \\
& \left.+\left|\boldsymbol{b}_{\zeta}^{K}\right|_{1, \tau}\left\|p-p_{H, h}\right\|_{0, \tau}\right\} \\
\preceq & \sum_{\tau \in \omega_{\zeta}}\left[h_{\tau}^{1 / 2}\left\|\boldsymbol{R}_{\tau}^{K}\right\|_{0, \tau}+h_{\tau}^{-1 / 2}\left|\boldsymbol{u}-\boldsymbol{u}_{H, h}\right|_{1, \tau}+h_{\tau}^{1 / 2}\left\|\boldsymbol{u}-\boldsymbol{u}_{H, h}\right\|_{0, \tau}\right. \\
& \left.+h_{\tau}^{-1 / 2}\left\|p-p_{H, h}\right\|_{0, \tau}\right]\left\|\boldsymbol{R}_{\zeta}^{K}\right\|_{0, \zeta}
\end{aligned}
$$

and then, from the regularity of the second level meshes, it holds

$$
h_{\zeta}^{1 / 2}\left\|\boldsymbol{R}_{\zeta}^{K}\right\|_{0, \zeta} \preceq \sum_{\tau \in \omega_{\zeta}}\left\{h_{\tau}\left\|\boldsymbol{R}_{\tau}^{K}\right\|_{0, \tau}+\left|\boldsymbol{u}-\boldsymbol{u}_{H, h}\right|_{1, \tau}+h_{\tau}\left\|\boldsymbol{u}-\boldsymbol{u}_{H, h}\right\|_{0, \tau}+\left\|p-p_{H, h}\right\|_{0, \tau}\right\} .
$$

For $\zeta \in \mathcal{E}_{h}^{K} \backslash \mathcal{E}_{0}^{K}$, from (2.13) and the definition of the norm $\|\cdot\|_{1 / 2, \partial K}$ in (2.11), and Theorem 2.4, we get

$$
\begin{aligned}
\left(\boldsymbol{R}_{\zeta}^{K}, \boldsymbol{b}_{\zeta}^{K}\right)_{\zeta}= & \sum_{\tau \in \omega_{\zeta}}\left\{-\left(\boldsymbol{R}_{\tau}^{K}, \boldsymbol{b}_{\zeta}^{K}\right)_{\tau}+\left(\nu \nabla\left(\boldsymbol{u}-\boldsymbol{u}_{H, h}\right), \nabla \boldsymbol{b}_{\zeta}^{K}\right)_{\tau}+\left(\gamma\left(\boldsymbol{u}-\boldsymbol{u}_{H, h}\right), \boldsymbol{b}_{\zeta}^{K}\right)_{\tau}\right. \\
& \left.-\left(\nabla \cdot \boldsymbol{b}_{\zeta}^{K}, p-p_{H, h}\right)_{\tau}\right\}+\left\langle\boldsymbol{\lambda}-\boldsymbol{\lambda}_{H, h}, \boldsymbol{b}_{\zeta}^{K}\right\rangle_{\partial K} \\
\preceq & \sum_{\tau \in \omega_{\zeta}}\left\{\left\|\boldsymbol{R}_{\tau}^{K}\right\|_{0, \tau}\left\|\boldsymbol{b}_{\zeta}^{K}\right\|_{0, \tau}+\left|\boldsymbol{u}-\boldsymbol{u}_{H, h}\right|_{1, \tau}\left|\boldsymbol{b}_{\zeta}^{K}\right|_{1, \tau}+\left\|\boldsymbol{u}-\boldsymbol{u}_{H, h}\right\|_{0, \tau}\left\|\boldsymbol{b}_{\zeta}^{K}\right\|_{0, \tau}\right. \\
& \left.+\left|\boldsymbol{b}_{\zeta}^{K}\right|_{1, \tau}\left\|p-p_{H, h}\right\|_{0, \tau}\right\}+\left\|\boldsymbol{\lambda}-\boldsymbol{\lambda}_{H, h}\right\|_{-1 / 2, \partial K}\left\|\boldsymbol{b}_{\zeta}^{K}\right\|_{1 / 2, \partial K} \\
\preceq & {\left[\sum _ { \tau \in \omega _ { \zeta } } \left\{h_{\tau}^{1 / 2}\left\|\boldsymbol{R}_{\tau}^{K}\right\|_{0, \tau}+h_{\tau}^{-1 / 2}\left|\boldsymbol{u}-\boldsymbol{u}_{H, h}\right|_{1, \tau}+h_{\tau}^{1 / 2}\left\|\boldsymbol{u}-\boldsymbol{u}_{H, h}\right\|_{0, \tau}\right.\right.} \\
& \left.\left.+h_{\tau}^{-1 / 2}\left\|p-p_{H, h}\right\|_{0, \tau}\right\}+h_{\zeta}^{-1 / 2}\left\|\boldsymbol{\lambda}-\boldsymbol{\lambda}_{H, h}\right\|_{-1 / 2, \partial K}\right]\left\|\boldsymbol{R}_{\zeta}^{K}\right\|_{0, \tau} .
\end{aligned}
$$


Now, from the regularity of the second-level meshes we get

$$
\begin{aligned}
h_{\zeta}^{1 / 2}\left\|\boldsymbol{R}_{\zeta}^{K}\right\|_{0, \zeta} \preceq & \sum_{\tau \in \omega_{\zeta}}\left\{h_{\tau}\left\|\boldsymbol{R}_{\tau}^{K}\right\|_{0, \tau}+\left|\boldsymbol{u}-\boldsymbol{u}_{H, h}\right|_{1, \tau}+h_{\tau}\left\|\boldsymbol{u}-\boldsymbol{u}_{H, h}\right\|_{0, \tau}\right. \\
& \left.+\left\|p-p_{H, h}\right\|_{0, \tau}\right\}+\left\|\boldsymbol{\lambda}-\boldsymbol{\lambda}_{H, h}\right\|_{-1 / 2, \partial K},
\end{aligned}
$$

and estimate (4.15) follows.

A first equivalence between the real error and the multiscale a posteriori estimator is presented in the next theorem.

Theorem 4.4. Let $(\boldsymbol{u}, p) \in \mathbf{V} \times Q$ and $\left(\boldsymbol{u}_{H, h}, p_{H, h}\right) \in \mathbf{V}_{h} \times Q_{h}$ be the solutions of continuous and discrete problems given by (3.6) and (3.9), respectively. Then we have that

$$
\left\|\boldsymbol{u}-\boldsymbol{u}_{H, h}\right\|_{\mathbf{v}}+\left\|p-p_{H, h}\right\|_{Q}+\left\|\boldsymbol{\lambda}-\boldsymbol{\lambda}_{H, h}\right\|_{\boldsymbol{\Lambda}} \preceq\left(\eta_{1}+\eta_{2}\right)+\text { h.o.t. }
$$

Moreover, for $F \in \mathcal{E}_{H}$, we have that

$$
\eta_{1, F} \preceq\left\|\boldsymbol{u}-\boldsymbol{u}_{H, h}\right\|_{\mathbf{V}, \omega_{F}} .
$$

Proof. We begin by proving the reliability of the estimator. From (3.6) and (3.20), it holds

$$
\begin{aligned}
\|(\boldsymbol{u}, p) & -\left(\boldsymbol{u}_{H, h}, p_{H, h}\right) \|_{\mathbf{V} \times Q} \\
& =\left\|\left(\boldsymbol{u}_{0}+T^{\boldsymbol{u}} \boldsymbol{\lambda}+\hat{T}^{\boldsymbol{u}} \boldsymbol{f}, T^{p} \boldsymbol{\lambda}+\hat{T}^{p} \boldsymbol{f}\right)-\left(\boldsymbol{u}_{0}^{H, h}+T_{h}^{\boldsymbol{u}} \boldsymbol{\lambda}_{H, h}+\hat{T}_{h}^{\boldsymbol{u}} \boldsymbol{f}, T_{h}^{p} \boldsymbol{\lambda}_{H, h}+\hat{T}_{h}^{p} \boldsymbol{f}\right)\right\|_{\mathbf{V} \times Q} \\
& \leq\left\|\boldsymbol{u}_{0}-\boldsymbol{u}_{0}^{H, h}\right\|_{\mathbf{v}}+\left\|\left(T^{\boldsymbol{u}}-T_{h}^{\boldsymbol{u}}\right) \boldsymbol{\lambda}_{H, h}+\left(\hat{T}^{\boldsymbol{u}}-\hat{T}_{h}^{\boldsymbol{u}}\right) \boldsymbol{f}\right\|_{\mathbf{V}}+\left\|T^{\boldsymbol{u}}\left(\boldsymbol{\lambda}-\boldsymbol{\lambda}_{H, h}\right)\right\|_{\mathbf{V}} \\
& +\left\|\left(T^{p}-T_{h}^{p}\right) \boldsymbol{\lambda}_{H, h}+\left(\hat{T}^{p}-\hat{T}_{h}^{p}\right) \boldsymbol{f}\right\|_{Q}+\left\|T^{p}\left(\boldsymbol{\lambda}-\boldsymbol{\lambda}_{H, h}\right)\right\|_{Q} .
\end{aligned}
$$

Furthermore, given $\boldsymbol{\mu}_{H} \in \boldsymbol{\Lambda}_{H}$ and using (3.5) and (3.13), we obtain

$$
\begin{aligned}
-\left(\boldsymbol{\mu}_{H}, \boldsymbol{u}_{0}-\boldsymbol{u}_{0}^{H, h}\right)_{\partial \mathcal{T}_{H}} & =\left(\boldsymbol{\mu}_{H}, T^{\boldsymbol{u}} \boldsymbol{\lambda}-T_{h}^{\boldsymbol{u}} \boldsymbol{\lambda}_{H, h}\right)_{\partial \mathcal{T}_{H}}+\left(\boldsymbol{\mu}_{H}, \hat{T}^{\boldsymbol{u}} \boldsymbol{f}-\hat{T}_{h}^{u} \boldsymbol{f}\right)_{\partial \mathcal{T}_{H}} \\
& =\left(\boldsymbol{\mu}_{H}, T^{\boldsymbol{u}}\left(\boldsymbol{\lambda}-\boldsymbol{\lambda}_{H, h}\right)\right)_{\partial \mathcal{T}_{H}}+\left(\boldsymbol{\mu}_{H},\left(T^{\boldsymbol{u}}-T_{h}^{\boldsymbol{u}}\right) \boldsymbol{\lambda}_{H, h}+\left(\hat{T}^{\boldsymbol{u}}-\hat{T}_{h}^{\boldsymbol{u}}\right) \boldsymbol{f}\right)_{\partial \mathcal{T}_{H}} \\
& \leq\left\|\boldsymbol{\mu}_{H}\right\|_{\boldsymbol{\Lambda}}\left\{\left\|T^{\boldsymbol{u}}\left(\boldsymbol{\lambda}-\boldsymbol{\lambda}_{H, h}\right)\right\|_{\mathbf{v}}+\left\|\left(T^{\boldsymbol{u}}-T_{h}^{\boldsymbol{u}}\right) \boldsymbol{\lambda}_{H, h}+\left(\hat{T}^{\boldsymbol{u}}-\hat{T}_{h}^{\boldsymbol{u}}\right) \boldsymbol{f}\right\|_{\mathbf{v}}\right\} .
\end{aligned}
$$

Consequently, we get

$$
\sup _{\boldsymbol{\mu}_{H} \in \boldsymbol{\Lambda}_{H}} \frac{-\left(\boldsymbol{\mu}_{H}, \boldsymbol{u}_{0}-\boldsymbol{u}_{0}^{H, h}\right) \partial \mathcal{T}_{H}}{\left\|\boldsymbol{\mu}_{H}\right\|_{\boldsymbol{\Lambda}}} \leq\left\|T^{\boldsymbol{u}}\left(\boldsymbol{\lambda}-\boldsymbol{\lambda}_{H, h}\right)\right\|_{\mathbf{v}}+\left\|\left(T^{\boldsymbol{u}}-T_{h}^{\boldsymbol{u}}\right) \boldsymbol{\lambda}_{H, h}+\left(\hat{T}^{\boldsymbol{u}}-\hat{T}_{h}^{\boldsymbol{u}}\right) \boldsymbol{f}\right\|_{\mathbf{v}}
$$

Now, since $\boldsymbol{u}_{0}-\boldsymbol{u}_{0}^{H, h} \in \mathbf{V}_{0}$, there exists a matrix function $\boldsymbol{\sigma}^{\star}$ such that each of its rows belong to the lowest order Raviart-Thomas space, satisfying

$$
\nabla \cdot \boldsymbol{\sigma}^{\star}=\boldsymbol{u}_{0}-\boldsymbol{u}_{0}^{H, h} \text { in } \Omega \quad \text { and } \quad\left\|\boldsymbol{\sigma}^{\star}\right\|_{\operatorname{div}, \Omega} \leq C\left\|\boldsymbol{u}_{0}-\boldsymbol{u}_{0}^{H, h}\right\|_{0, \Omega} .
$$

Defining $\boldsymbol{\mu}^{\star}:=\boldsymbol{\sigma}^{\star} \boldsymbol{n} \in \boldsymbol{\Lambda}_{H}$, and using (4.20), it holds

$$
\begin{aligned}
\sup _{\boldsymbol{\mu}_{H} \in \boldsymbol{\Lambda}_{H}} \frac{-\left(\boldsymbol{\mu}_{H}, \boldsymbol{u}_{0}-\boldsymbol{u}_{0}^{H, h}\right)_{\partial \mathcal{T}_{H}}}{\left\|\boldsymbol{\mu}_{H}\right\|_{\boldsymbol{\Lambda}}} & \geq \frac{\left(\boldsymbol{\mu}^{\star}, \boldsymbol{u}_{0}-\boldsymbol{u}_{0}^{H, h}\right)_{\partial \mathcal{T}_{H}}}{\left\|\boldsymbol{\mu}^{\star}\right\|_{\boldsymbol{\Lambda}}} \geq \frac{\left(\boldsymbol{\sigma}^{\star} \boldsymbol{n}, \boldsymbol{u}_{0}-\boldsymbol{u}_{0}^{H, h}\right)_{\partial \mathcal{T}_{H}}}{\left\|\boldsymbol{\sigma}^{\star}\right\|_{\operatorname{div}, \Omega}} \\
& =\frac{\left(\nabla \cdot \boldsymbol{\sigma}^{\star}, \boldsymbol{u}_{0}-\boldsymbol{u}_{0}^{H, h}\right)_{\partial \mathcal{T}_{H}}}{\left\|\boldsymbol{\sigma}^{\star}\right\|_{\operatorname{div}, \Omega}} \geq C\left\|\boldsymbol{u}_{0}-\boldsymbol{u}_{0}^{H, h}\right\|_{0, \Omega} \geq C\left\|\boldsymbol{u}_{0}-\boldsymbol{u}_{0}^{H, h}\right\|_{\mathbf{v}} .
\end{aligned}
$$

Using (4.21) in (4.19), and replacing the result in (4.18), and from Lemmas 4.1, using (A.13) and Lemmas A.2 and A.3 in the appendix, estimate (4.16) follows.

As for the efficiency of the a posteriori error estimator, we first recall the following scaling property (cf. [2, Theorem 3.10]): Given $v \in H^{1}(K)$, we have that

$$
\|v\|_{0, \partial K} \preceq\left(\frac{1}{H_{K}}\|v\|_{0, K}^{2}+H_{K}\|\nabla v\|_{0, K}^{2}\right)^{1 / 2} .
$$

Since $\boldsymbol{R}_{F} \in L^{2}(F)^{d}$,

$$
\left\|\boldsymbol{R}_{F}\right\|_{0, F}^{2} \leq 2\left(\boldsymbol{R}_{F}, \llbracket \boldsymbol{u}-\boldsymbol{u}_{H, h} \rrbracket\right)_{F} \leq 2\left\|\boldsymbol{R}_{F}\right\|_{0, F}\left\|\llbracket \boldsymbol{u}-\boldsymbol{u}_{H, h} \rrbracket\right\|_{0, F}
$$


and using (4.22) and the mesh regularity, it holds

$$
\begin{aligned}
\left\|\boldsymbol{R}_{F}\right\|_{0, F} & \leq 2\left\|\llbracket \boldsymbol{u}-\boldsymbol{u}_{H, h} \rrbracket\right\|_{0, F} \\
& \preceq \sum_{K \in \omega_{F}}\left(H_{K}^{-1}\left\|\boldsymbol{u}-\boldsymbol{u}_{H, h}\right\|_{0, K}^{2}+H_{K}\left\|\nabla\left(\boldsymbol{u}-\boldsymbol{u}_{H, h}\right)\right\|_{0, K}^{2}\right)^{1 / 2} \\
& \preceq H_{F}^{1 / 2} \sum_{K \in \omega_{F}}\left(H_{K}^{-2}\left\|\boldsymbol{u}-\boldsymbol{u}_{H, h}\right\|_{0, K}^{2}+\left\|\nabla\left(\boldsymbol{u}-\boldsymbol{u}_{H, h}\right)\right\|_{0, K}^{2}\right)^{1 / 2} \\
& \preceq H_{F}^{1 / 2}\left\|\boldsymbol{u}-\boldsymbol{u}_{H, h}\right\|_{\mathbf{V}, \omega_{F}},
\end{aligned}
$$

and the estimate (4.17) follows.

We are ready to present the main result of this section.

Theorem 4.5. Let $(\boldsymbol{u}, p, \boldsymbol{\lambda}) \in \mathbf{V} \times Q \times \boldsymbol{\Lambda}$ and $\left(\boldsymbol{u}_{H, h}, p_{H, h}, \boldsymbol{\lambda}_{H, h}\right) \in \mathbf{V}_{h} \times Q_{h} \times \boldsymbol{\Lambda}_{H}$ be the solutions of continuous and discrete problems, characterized through (3.6) and (3.9), respectively. Then, we have that

$$
\left\|\boldsymbol{u}-\boldsymbol{u}_{H, h}\right\|_{\mathbf{v}}+\left\|p-p_{H, h}\right\|_{Q}+\|\boldsymbol{\lambda}-\boldsymbol{\lambda}\|_{\boldsymbol{\Lambda}} \preceq\left(\eta_{1}+\eta_{2}\right)+\text { h.o.t. }
$$

Moreover, given $K \in \mathcal{T}_{H}$ and $F \in \mathcal{E}_{H}$, we have

$$
\eta_{1, F} \preceq\left\|\boldsymbol{u}-\boldsymbol{u}_{H, h}\right\|_{\mathbf{V}, \omega_{F}}
$$

and

$$
\eta_{2, K} \preceq\left\|\boldsymbol{u}-\boldsymbol{u}_{H, h}\right\|_{\mathbf{V}(K)}+\left\|p-p_{H, h}\right\|_{Q(K)}+\left\|\boldsymbol{\lambda}-\boldsymbol{\lambda}_{H, h}\right\|_{-1 / 2, \partial K} .
$$

Proof. The result is straightforward from Theorem 4.3 and Theorem 4.4.

\section{NumericAl RESUlts}

Using the definitions of the error estimators $\eta_{1}$ and $\eta_{2}$ given in (4.3) and (4.6), respectively, we validate two adaptive refinement algorithms: a classical procedure based on refining the elements of the first level mesh, and a new one based on refining faces which keeps the topology of the first-level mesh untouched.

5.1. First strategy: Adaptivity by elements. For all $K \in \mathcal{T}_{H}$, we define

$$
\eta_{K}:=\eta_{2, K}+\sum_{F \in \mathcal{E}_{H} \cap \partial K} \eta_{1, F} .
$$

The adaptive mesh generation strategy is given in the Algorithm 1.

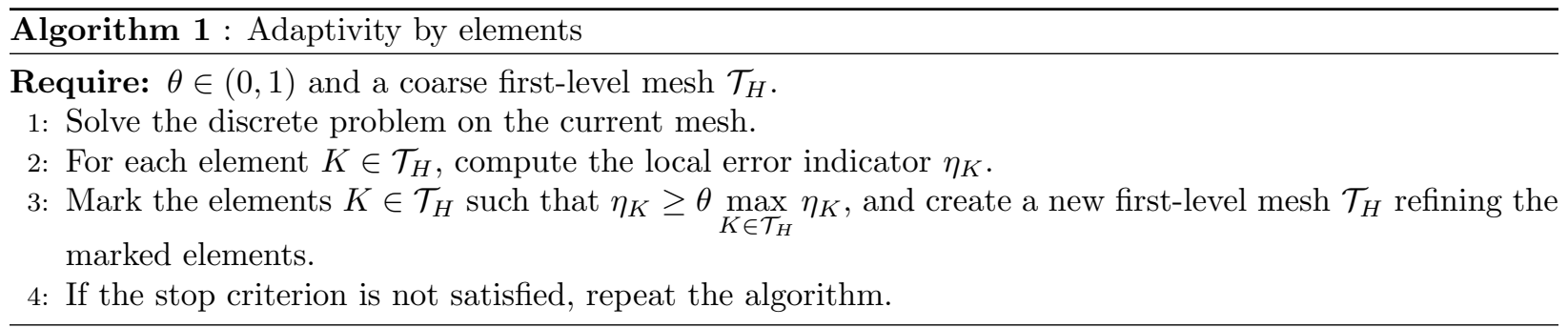

Note that Algorithm 1 does not induce local mesh refinement in the second-level sub-meshes. This fact is supported by the numerical experiments that show that second-level meshes may consist only of one element when the first-level mesh diameter goes to zero. 
5.2. Second strategy: Adaptivity by faces. For all $F \in \mathcal{E}_{H}$, we define

$$
\eta_{F}:=\eta_{1, F}+\sum_{K \in \omega_{F}} \eta_{2, K}
$$

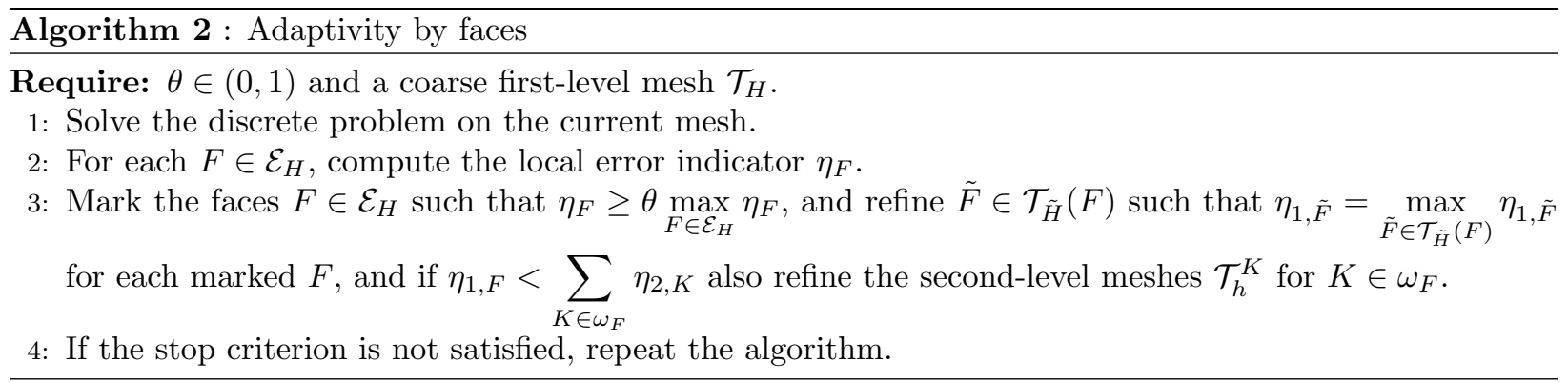

Owing to Algorithm 2, the first-level mesh does not change as the adaptive process runs unlike in the classical mesh adaptation described in Algorithm 1. As a result, only the local problems associated with the elements "touched" by the estimator need to be revisited. This fact leads to a few extra entries go into the global system. Algorithm 2 is particularly attractive for use with real three-dimensional problems since it dramatically decreases the computational cost involved in the adaptive procedure, and avoids threedimensional global re-meshing.

5.3. An example with analytical solution. In this example, we consider a Stokes problem with viscosity $\nu=1$ and $\nu=10^{-2}$ on the unit square domain $\Omega$. We chose $\boldsymbol{f}$ and $\boldsymbol{g}$ such that the exact solution $\boldsymbol{u}=\left(u_{1}, u_{2}\right)$ is given by $u_{1}(x, y):=256 y^{2}(y-1)^{2} x(x-1)(2 x-1)$ and $u_{2}(x, y):=-u_{1}(y, x)$, and $p(x, y):=150(x-0.5)(y-0.5)$.

A typical quantity to measure the quality of an error estimator, is the so-called effectivity index, $E_{i}$, defined by $E_{i}=\frac{\eta}{\left\|\left(\boldsymbol{u}-\boldsymbol{u}_{H, h}, p-p_{H, h}\right)\right\|_{\mathbf{v} \times Q}}$. In tables 1, 2, 3 and 4 we tabulate this index for some different values of $H$ and using one element as second-level meshes.

We observe that $\eta_{1}$ and $\eta_{2}$ have, in general, equivalent rates of convergence (see Tables 1 and 3). However, if one chooses $h=H^{2}$ then $\eta_{2}$ achieves a higher order of convergence when compare to $\eta_{1}$ (see Tables 2 and 4). This is in accordance with the theory and was announced in Remark 4.

\begin{tabular}{c|c|cc|cc|cc|c}
\hline \hline & & $\left\|\left(\boldsymbol{u}-\boldsymbol{u}_{H, h}, p-p_{H, h}\right)\right\| \mathbf{v} \times Q$ & \multicolumn{2}{|c|}{$\eta_{1}$} & \multicolumn{2}{|c}{$\eta_{2}$} & \\
$l$ & $H$ & error & rate & error & rate & value & rate & $E_{i}$ \\
\hline \hline & $1 / 2$ & $5.001 \mathrm{E}-01$ & - & $1.091 \mathrm{E}-01$ & - & $9.548 \mathrm{E}+00$ & - & 0.218 \\
& $1 / 4$ & $6.947 \mathrm{E}-02$ & 2.848 & $1.437 \mathrm{E}-02$ & 2.925 & $1.276 \mathrm{E}+00$ & 2.904 & 0.207 \\
& $1 / 8$ & $8.663 \mathrm{E}-03$ & 3.003 & $1.780 \mathrm{E}-03$ & 3.013 & $1.557 \mathrm{E}-01$ & 3.034 & 0.205 \\
& $1 / 16$ & $1.079 \mathrm{E}-03$ & 3.006 & $2.254 \mathrm{E}-04$ & 2.981 & $1.925 \mathrm{E}-02$ & 3.016 & 0.209 \\
& $1 / 32$ & $1.345 \mathrm{E}-04$ & 3.003 & $2.858 \mathrm{E}-05$ & 2.980 & $2.397 \mathrm{E}-03$ & 3.006 & 0.212 \\
\hline & $1 / 2$ & $8.001 \mathrm{E}-01$ & - & $4.747 \mathrm{E}-01$ & - & $5.708 \mathrm{E}+00$ & - & 0.593 \\
& $1 / 4$ & $3.516 \mathrm{E}-01$ & 1.186 & $2.000 \mathrm{E}-01$ & 1.247 & $1.818 \mathrm{E}+00$ & 1.651 & 0.569 \\
1 & $1 / 8$ & $8.642 \mathrm{E}-02$ & 2.024 & $5.168 \mathrm{E}-02$ & 1.953 & $4.640 \mathrm{E}-01$ & 1.970 & 0.598 \\
& $1 / 16$ & $2.144 \mathrm{E}-02$ & 2.011 & $1.335 \mathrm{E}-02$ & 1.953 & $1.135 \mathrm{E}-01$ & 2.031 & 0.623 \\
& $1 / 32$ & $5.365 \mathrm{E}-03$ & 1.998 & $3.418 \mathrm{E}-03$ & 1.965 & $2.787 \mathrm{E}-02$ & 2.027 & 0.637 \\
\hline & $1 / 2$ & $9.719 \mathrm{E}+00$ & - & $6.203 \mathrm{E}+00$ & - & $6.187 \mathrm{E}+00$ & - & 0.638 \\
& $1 / 4$ & $4.762 \mathrm{E}+00$ & 1.029 & $3.503 \mathrm{E}+00$ & 0.825 & $2.151 \mathrm{E}+00$ & 1.524 & 0.736 \\
0 & $1 / 8$ & $2.339 \mathrm{E}+00$ & 1.026 & $2.001 \mathrm{E}+00$ & 0.808 & $1.040 \mathrm{E}+00$ & 1.048 & 0.855 \\
& $1 / 16$ & $1.148 \mathrm{E}+00$ & 1.027 & $1.064 \mathrm{E}+00$ & 0.911 & $5.189 \mathrm{E}-01$ & 1.003 & 0.927 \\
& $1 / 32$ & $5.686 \mathrm{E}-01$ & 1.014 & $5.456 \mathrm{E}-01$ & 0.964 & $2.594 \mathrm{E}-01$ & 1.001 & 0.960 \\
\hline \hline
\end{tabular}

TABLE 1. History of convergence for the Stokes problem with $\nu=1, \boldsymbol{u}_{H, h} \in \mathbb{P}^{3}\left(\mathcal{T}_{H}\right)^{2}$, $p_{h} \in \mathbb{P}^{3}\left(\mathcal{T}_{H}\right)$ and $\boldsymbol{\lambda}_{H, h} \in \boldsymbol{\Lambda}_{l}^{1}$. 


\begin{tabular}{c|c|cc|cc|cc|c}
\hline \hline & & $\left\|\left(\boldsymbol{u}-\boldsymbol{u}_{H, h}, p-p_{H, h}\right)\right\|_{\mathbf{V} \times Q}$ & \multicolumn{2}{|c|}{$\eta_{1}$} & \multicolumn{2}{|c}{$\eta_{2}$} & \\
$l$ & $H$ & error & rate & error & rate & value & rate & $E_{i}$ \\
\hline \hline & $1 / 2$ & $2.993 \mathrm{E}-01$ & - & $6.850 \mathrm{E}-02$ & - & $1.562 \mathrm{E}+00$ & - & 0.229 \\
& $1 / 4$ & $3.250 \mathrm{E}-02$ & 3.203 & $9.136 \mathrm{E}-03$ & 2.906 & $4.013 \mathrm{E}-02$ & 5.282 & 0.281 \\
2 & $1 / 8$ & $3.701 \mathrm{E}-03$ & 3.135 & $1.171 \mathrm{E}-03$ & 2.964 & $1.407 \mathrm{E}-03$ & 4.834 & 0.316 \\
& $1 / 16$ & $4.480 \mathrm{E}-04$ & 3.046 & $1.515 \mathrm{E}-04$ & 2.951 & $5.545 \mathrm{E}-05$ & 4.665 & 0.338 \\
\hline \hline & $1 / 2$ & $7.746 \mathrm{E}-01$ & - & $3.523 \mathrm{E}-01$ & - & $1.803 \mathrm{E}+00$ & - & 0.455 \\
& $1 / 4$ & $3.564 \mathrm{E}-01$ & 1.120 & $1.487 \mathrm{E}-01$ & 1.244 & $1.589 \mathrm{E}-01$ & 3.504 & 0.417 \\
1 & $1 / 8$ & $8.783 \mathrm{E}-02$ & 2.021 & $3.854 \mathrm{E}-02$ & 1.948 & $1.321 \mathrm{E}-02$ & 3.589 & 0.439 \\
& $1 / 16$ & $2.178 \mathrm{E}-02$ & 2.012 & $9.941 \mathrm{E}-03$ & 1.955 & $1.054 \mathrm{E}-03$ & 3.647 & 0.456 \\
\hline \hline & $1 / 2$ & $9.720 \mathrm{E}+00$ & - & $4.387 \mathrm{E}+00$ & - & $1.370 \mathrm{E}+00$ & - & 0.451 \\
& $1 / 4$ & $4.764 \mathrm{E}+00$ & 1.029 & $2.477 \mathrm{E}+00$ & 0.825 & $2.034 \mathrm{E}-01$ & 2.752 & 0.520 \\
0 & $1 / 8$ & $2.340 \mathrm{E}+00$ & 1.025 & $1.415 \mathrm{E}+00$ & 0.808 & $3.354 \mathrm{E}-02$ & 2.600 & 0.605 \\
& $1 / 16$ & $1.148 \mathrm{E}+00$ & 1.027 & $7.524 \mathrm{E}-01$ & 0.911 & $5.439 \mathrm{E}-03$ & 2.625 & 0.655 \\
\hline \hline
\end{tabular}

TABLE 2. History convergence for the Stokes problem with $\nu=1, \boldsymbol{u}_{H, h} \in \mathbb{P}^{3}\left(\mathcal{T}_{H}\right)^{2}, p_{h} \in$ $\mathbb{P}^{3}\left(\mathcal{T}_{H}\right)$ and $\boldsymbol{\lambda}_{H, h} \in \boldsymbol{\Lambda}_{l}^{1}$. Here $h=H^{2}$.

\begin{tabular}{c|c|cc|cc|cc|c}
\hline \hline & & $\left\|\left(\boldsymbol{u}-\boldsymbol{u}_{H, h}, p-p_{H, h}\right)\right\|_{\mathbf{V} \times Q}$ & \multicolumn{2}{|c|}{$\eta_{1}$} & \multicolumn{2}{|c}{$\eta_{2}$} & \\
$l$ & $H$ & error & rate & error & rate & value & rate & $E_{i}$ \\
\hline \hline & $1 / 2$ & $4.365 \mathrm{E}-01$ & - & $7.712 \mathrm{E}-02$ & - & $2.306 \mathrm{E}-01$ & - & 0.705 \\
& $1 / 4$ & $6.190 \mathrm{E}-02$ & 2.818 & $1.016 \mathrm{E}-02$ & 2.925 & $2.776 \mathrm{E}-02$ & 3.055 & 0.613 \\
2 & $1 / 8$ & $7.709 \mathrm{E}-03$ & 3.005 & $1.259 \mathrm{E}-03$ & 3.013 & $3.255 \mathrm{E}-03$ & 3.092 & 0.586 \\
& $1 / 16$ & $9.593 \mathrm{E}-04$ & 3.006 & $1.594 \mathrm{E}-04$ & 2.981 & $3.981 \mathrm{E}-04$ & 3.032 & 0.581 \\
& $1 / 32$ & $1.197 \mathrm{E}-04$ & 3.003 & $2.021 \mathrm{E}-05$ & 2.980 & $4.943 \mathrm{E}-05$ & 3.009 & 0.582 \\
\hline & $1 / 2$ & $5.342 \mathrm{E}+01$ & - & $2.717 \mathrm{E}+01$ & - & $8.219 \mathrm{E}+00$ & - & 0.662 \\
& $1 / 4$ & $1.350 \mathrm{E}+01$ & 1.985 & $6.936 \mathrm{E}+00$ & 1.970 & $2.106 \mathrm{E}+00$ & 1.964 & 0.670 \\
1 & $1 / 8$ & $3.388 \mathrm{E}+00$ & 1.994 & $1.746 \mathrm{E}+00$ & 1.990 & $5.313 \mathrm{E}-01$ & 1.987 & 0.672 \\
& $1 / 16$ & $8.483 \mathrm{E}-01$ & 1.998 & $4.378 \mathrm{E}-01$ & 1.996 & $1.333 \mathrm{E}-01$ & 1.995 & 0.673 \\
& $1 / 32$ & $2.122 \mathrm{E}-01$ & 1.999 & $1.096 \mathrm{E}-01$ & 1.998 & $3.338 \mathrm{E}-02$ & 1.998 & 0.674 \\
\hline & $1 / 2$ & $4.889 \mathrm{E}+02$ & - & $2.842 \mathrm{E}+02$ & - & $6.531 \mathrm{E}+00$ & - & 0.595 \\
& $1 / 4$ & $2.793 \mathrm{E}+02$ & 0.808 & $1.828 \mathrm{E}+02$ & 0.637 & $3.267 \mathrm{E}+00$ & 0.999 & 0.666 \\
0 & $1 / 8$ & $1.505 \mathrm{E}+02$ & 0.892 & $1.097 \mathrm{E}+02$ & 0.736 & $1.634 \mathrm{E}+00$ & 1.000 & 0.740 \\
& $1 / 16$ & $7.748 \mathrm{E}+01$ & 0.958 & $5.918 \mathrm{E}+01$ & 0.891 & $8.170 \mathrm{E}-01$ & 1.000 & 0.774 \\
& $1 / 32$ & $3.913 \mathrm{E}+01$ & 0.985 & $3.048 \mathrm{E}+01$ & 0.957 & $4.085 \mathrm{E}-01$ & 1.000 & 0.789 \\
\hline \hline
\end{tabular}

TABLE 3. History of convergence for the Stokes problem with $\nu=10^{-2}, \boldsymbol{u}_{H, h} \in \mathbb{P}^{3}\left(\mathcal{T}_{H}\right)^{2}$, $p_{h} \in \mathbb{P}^{3}\left(\mathcal{T}_{H}\right)$ and $\boldsymbol{\lambda}_{H, h} \in \boldsymbol{\Lambda}_{l}^{1}$. 


\begin{tabular}{c|c|cc|cc|cc|c}
\hline \hline & & $\left\|\left(\boldsymbol{u}-\boldsymbol{u}_{H, h}, p-p_{H, h}\right)\right\|_{\mathbf{V} \times Q}$ & \multicolumn{2}{|c|}{$\eta_{1}$} & \multicolumn{2}{|c|}{$\eta_{2}$} & \\
$l$ & $H$ & error & rate & error & rate & value & rate & $E_{i}$ \\
\hline \hline & $1 / 2$ & $2.254 \mathrm{E}-01$ & - & $6.850 \mathrm{E}-02$ & - & $3.861 \mathrm{E}-02$ & - & 0.475 \\
& $1 / 4$ & $2.663 \mathrm{E}-02$ & 3.082 & $9.136 \mathrm{E}-03$ & 2.906 & $1.090 \mathrm{E}-03$ & 5.146 & 0.384 \\
2 & $1 / 8$ & $3.147 \mathrm{E}-03$ & 3.081 & $1.171 \mathrm{E}-03$ & 2.964 & $3.866 \mathrm{E}-05$ & 4.818 & 0.384 \\
& $1 / 16$ & $3.878 \mathrm{E}-04$ & 3.020 & $1.515 \mathrm{E}-04$ & 2.951 & $1.520 \mathrm{E}-06$ & 4.668 & 0.395 \\
\hline \hline & $1 / 2$ & $5.147 \mathrm{E}+01$ & - & $2.932 \mathrm{E}+01$ & - & $3.404 \mathrm{E}+00$ & - & 0.636 \\
& $1 / 4$ & $1.308 \mathrm{E}+01$ & 1.976 & $7.526 \mathrm{E}+00$ & 1.962 & $2.941 \mathrm{E}-01$ & 3.533 & 0.598 \\
1 & $1 / 8$ & $3.288 \mathrm{E}+00$ & 1.992 & $1.895 \mathrm{E}+00$ & 1.990 & $2.404 \mathrm{E}-02$ & 3.613 & 0.584 \\
& $1 / 16$ & $8.237 \mathrm{E}-01$ & 1.997 & $4.750 \mathrm{E}-01$ & 1.996 & $1.945 \mathrm{E}-03$ & 3.627 & 0.579 \\
\hline \hline & $1 / 2$ & $4.890 \mathrm{E}+02$ & - & $2.843 \mathrm{E}+02$ & - & $2.561 \mathrm{E}+00$ & - & 0.587 \\
& $1 / 4$ & $2.795 \mathrm{E}+02$ & 0.807 & $1.826 \mathrm{E}+02$ & 0.638 & $4.074 \mathrm{E}-01$ & 2.652 & 0.655 \\
0 & $1 / 8$ & $1.506 \mathrm{E}+02$ & 0.892 & $1.096 \mathrm{E}+02$ & 0.736 & $6.476 \mathrm{E}-02$ & 2.653 & 0.728 \\
& $1 / 16$ & $7.752 \mathrm{E}+01$ & 0.958 & $5.913 \mathrm{E}+01$ & 0.890 & $1.039 \mathrm{E}-02$ & 2.640 & 0.763 \\
\hline \hline
\end{tabular}

TABLE 4. History of convergence for the Stokes problem with $\nu=10^{-2}, \boldsymbol{u}_{H, h} \in \mathbb{P}^{3}\left(\mathcal{T}_{H}\right)^{2}$, $p_{h} \in \mathbb{P}^{3}\left(\mathcal{T}_{H}\right)$ and $\boldsymbol{\lambda}_{H, h} \in \boldsymbol{\Lambda}_{l}^{1}$. Here $h=H^{2}$.

5.4. The 2D lid-driven cavity problem. We consider the well-known 2D cavity problem for the Stokes $(\gamma=\mathbf{0})$ and Brinkman $\left(\boldsymbol{\gamma}=10^{4} \mathbf{I}\right)$ problems with $\nu=1$. In Figures 2 and 3, we show the meshes obtained by the two versions of the adaptive algorithms looking for $\boldsymbol{u}_{h} \in \mathbb{P}^{2}(K)^{2}, p_{h} \in \mathbb{P}^{2}(K)$ and $\boldsymbol{\lambda}_{H, h} \in \boldsymbol{\Lambda}_{0}$. Higher polynomial degrees does not change conclusions and then they are not presented. 

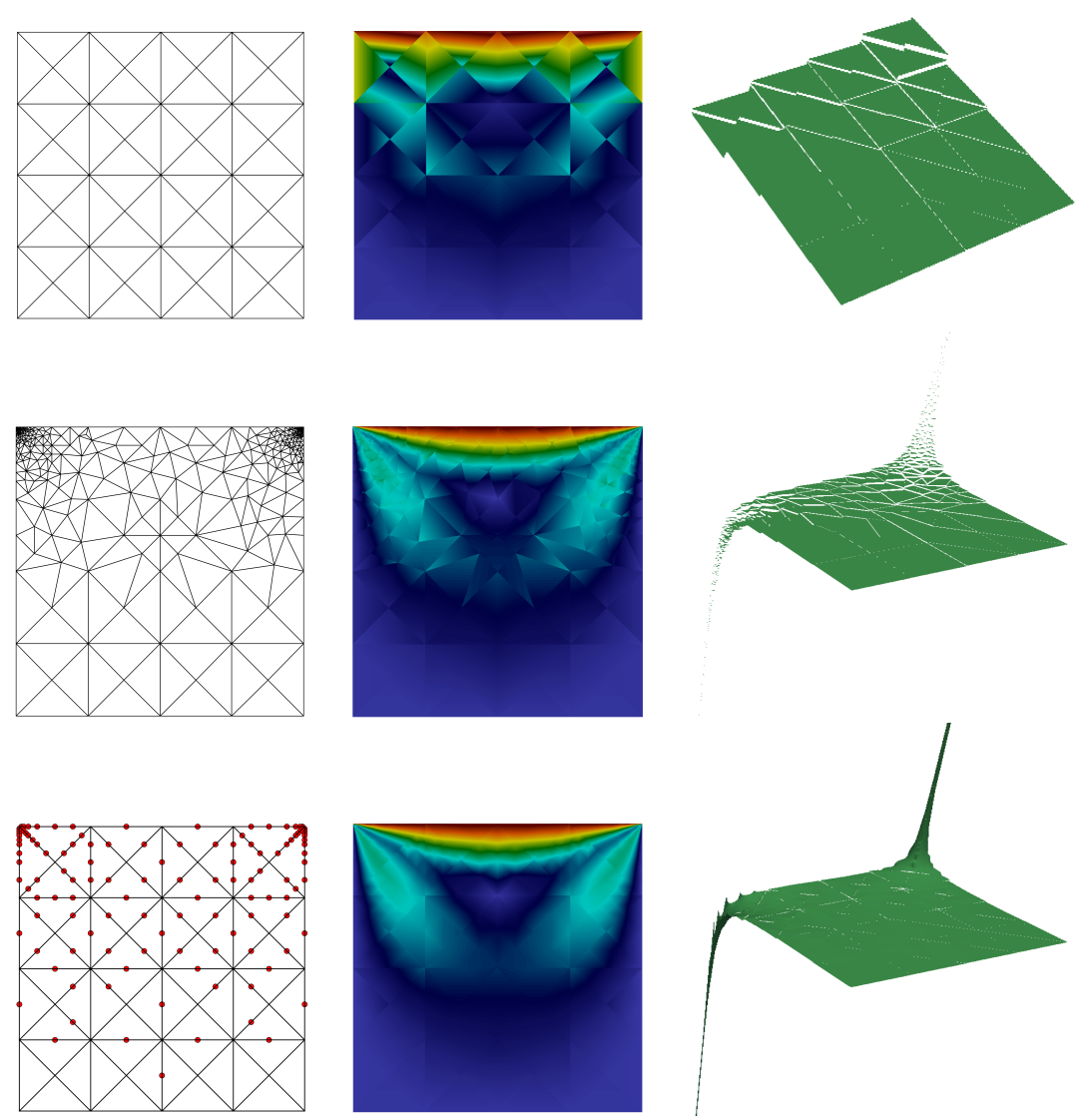

FiguRE 2. Lid-driven cavity problem for the Stokes equation with $\boldsymbol{u}_{h} \in \mathbb{P}^{2}(K)^{2}, p_{h} \in$ $\mathbb{P}^{2}(K), \boldsymbol{\lambda}_{H, h} \in \boldsymbol{\Lambda}_{0}$ and $\nu=1$. Second and third rows correspond, respectively, to the final step of the adaptive procedure by elements (6,216 dof) and adaptive procedure by faces (912 dof), and the first row shows the start step in both adaptive procedures. Second and third columns represent the magnitude of the velocity and pressure, respectively. 

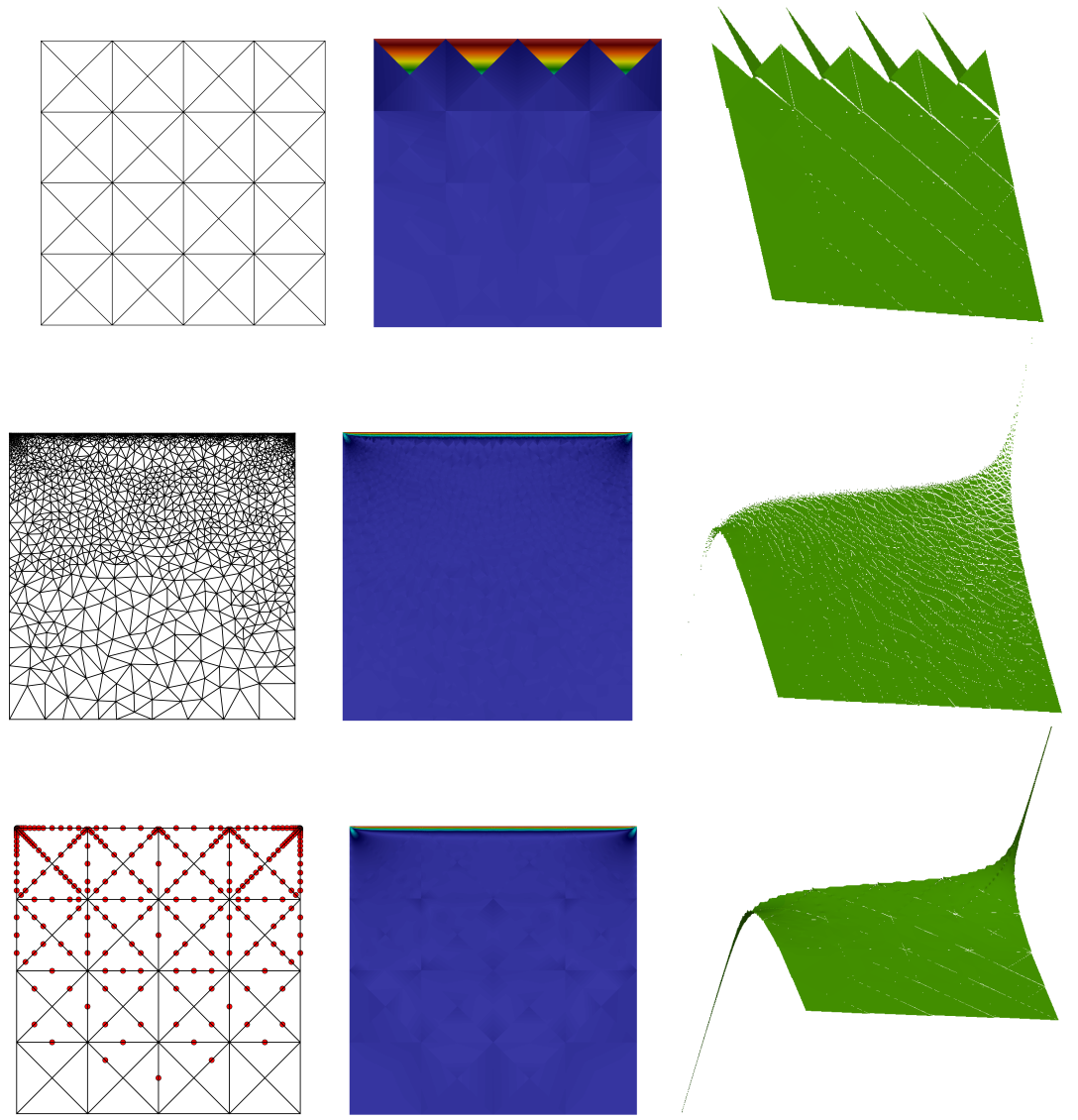

Figure 3. Lid-driven cavity problem for the Brinkman equation with $\boldsymbol{u}_{h} \in \mathbb{P}^{2}(K)^{2}, p_{h} \in$ $\mathbb{P}^{2}(K), \boldsymbol{\lambda}_{H, h} \in \boldsymbol{\Lambda}_{0}, \nu=1$ and $\boldsymbol{\gamma}=10^{4} \mathbf{I}$. Second and third rows correspond, respectively, to the final step of the adaptive procedure by elements (32,337 dof) and adaptive procedure by faces (786 dof), and the first row shows the start step in both adaptive procedures. Second and third columns represent the magnitude of the velocity and pressure, respectively.

As expected, in the cavity problem for Stokes and Brinkman, the adaptive algorithms refine the upper part of the domain, where the solution has boundary layers and/or singularities. Note that Algorithm 2 requires less degrees of freedom than Algorithm 1 to get good numerical results.

5.5. A highly heterogeneous case. This numerical test illustrates the capacity of the MHM method to simulate fluid flow in a highly heterogeneous porous media. The domain is $\Omega:=] 0,1200[\times] 0,2200[$ with boundary conditions given in Figure 4. We adopt a heterogeneous isotropic coefficient $\gamma$ obtained from the 85th layer of the SPE10 project [15] (second dataset). The domain represents a quite realistic prototype of a reservoir. Here, we adopt the following version of the Brinkman model

$$
-\nu \Delta \boldsymbol{u}+\boldsymbol{\gamma} \boldsymbol{u}+\nabla p=\mathbf{0} \quad \text { in } \Omega, \quad \nabla \cdot \boldsymbol{u}=0 \quad \text { in } \Omega
$$

with $\nu=0.3$ and $\gamma=(0.3 / \kappa) \mathbf{I}$, where $\mathbf{I}$ stands for the identity tensor. The permeability $\kappa$ is depicted in Figure 4. The reference solution is calculated using the USFEM scheme of [10] using a uniform mesh of $1,081,344$ triangles. The $\mathbb{P}_{1}(\tau)^{d} \times \mathbb{P}_{1}(\tau)$ element is adopt to approximate the velocity and pressure variables. As such, the total number of degrees of freedom is 1,625, 283. As for the MHM solution, we select $\boldsymbol{u}_{h} \in \mathbb{P}^{3}(K)^{2}, p_{h} \in \mathbb{P}^{3}(K)$ and $\boldsymbol{\lambda}_{H, h} \in \boldsymbol{\Lambda}_{1}$. 

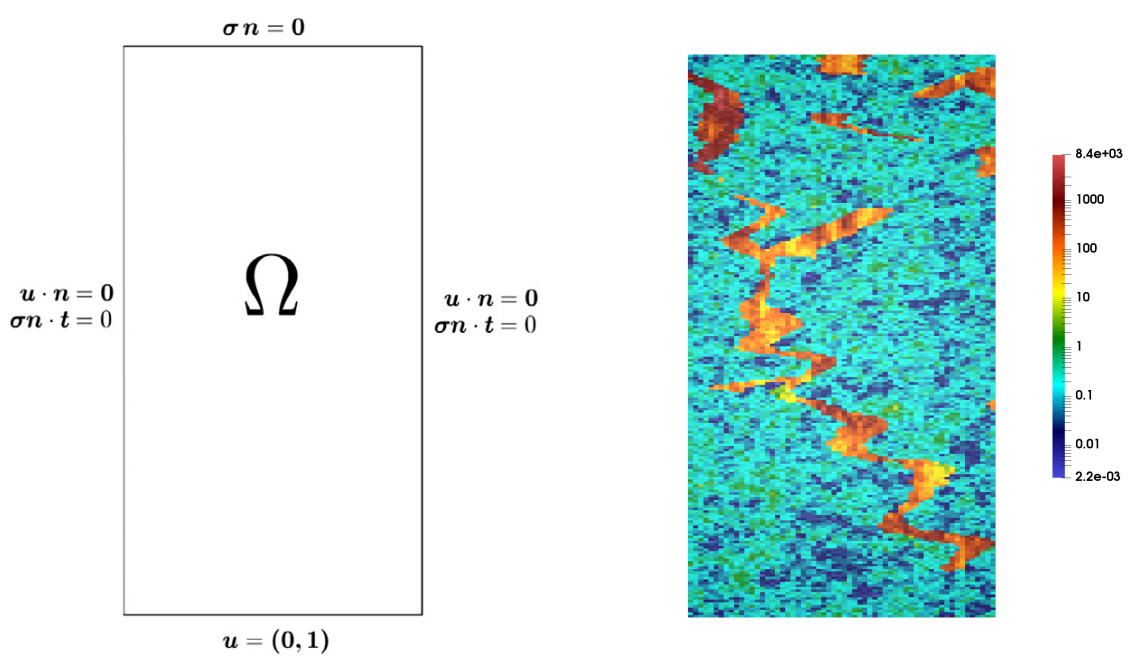

FIGURE 4. Boundary conditions (left) and the permeability $\kappa$ (right) in logarithmic scale.
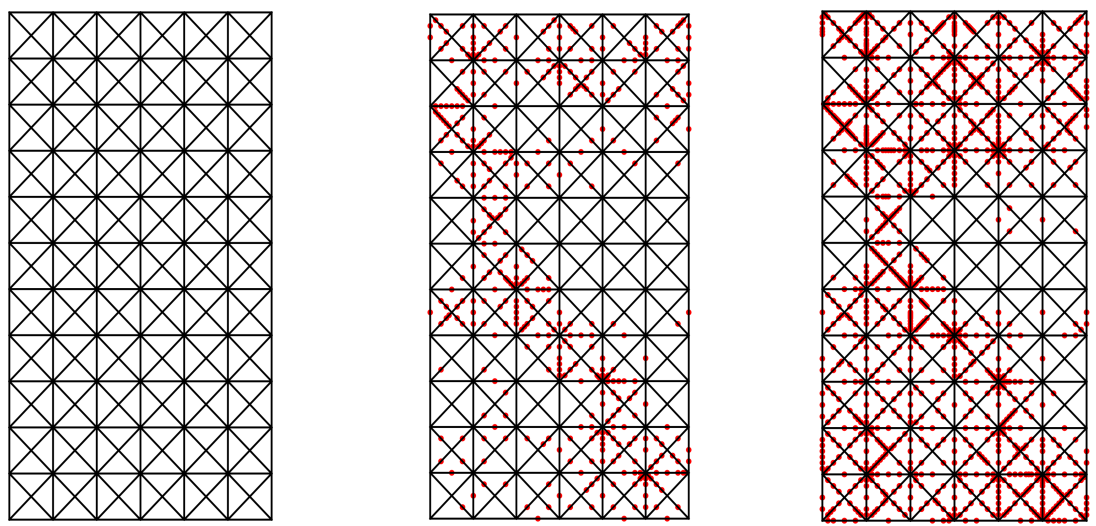

FiguRE 5. Initial mesh (left) with 1,652 dof, an intermediate adapted mesh (center) with 3,352 dof and the final adapted mesh (right) with 7,800 dof.
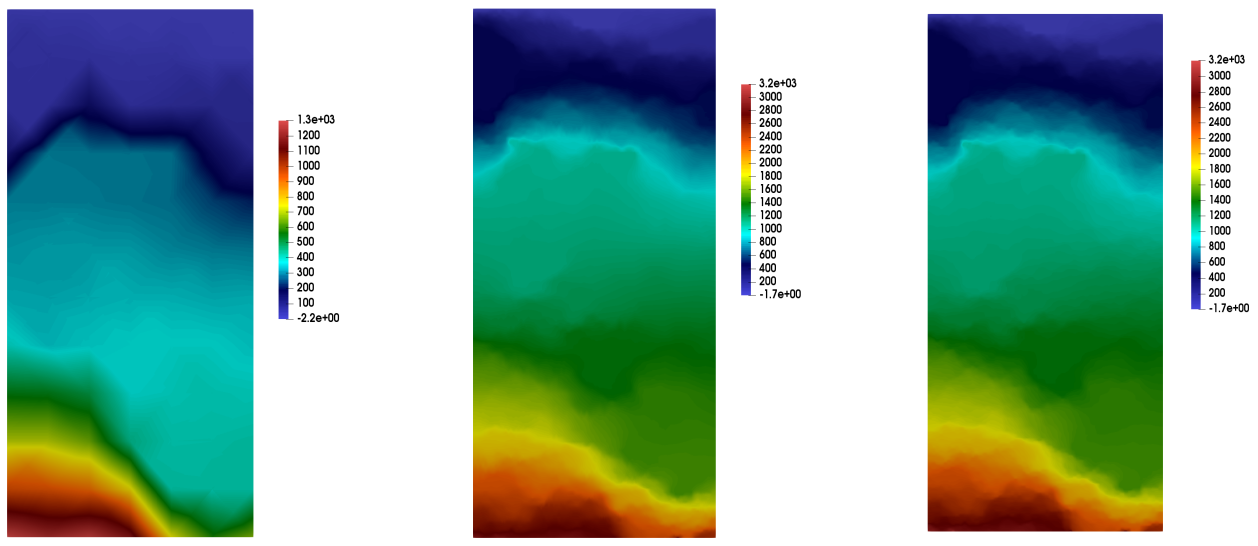

FiguRE 6. Isolines of the pressure corresponding (from left to right) to the solution using the initial mesh (1,652 dofs), the adaptive one (7,800 dofs), and the reference solution $(1,625,283$ dofs), respectively. 

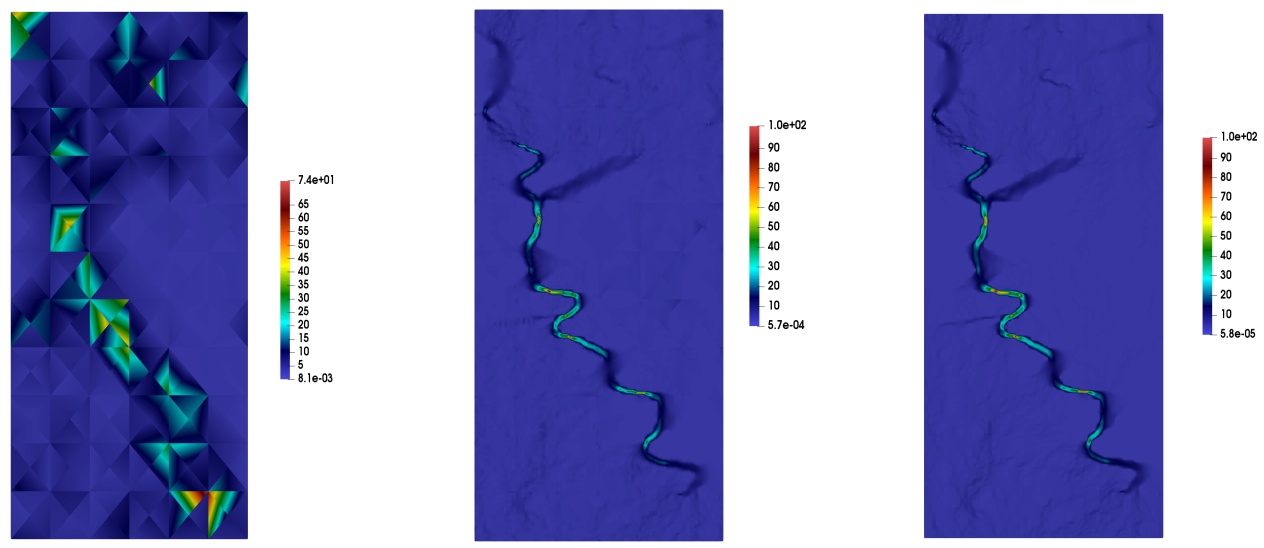

Figure 7. Magnitude of velocity corresponding (from left to right) to the solution using the initial mesh (1,652 dofs), the adaptive one (7,800 dofs), and the reference solution $(1,625,283$ dofs), respectively.

We observe that the solution of the MHM method using the adaptive strategy given by the Algorithm 2 is very close to the reference solution, and captures the heterogeneity of the reservoir clearly with the advantage of using considerably less degrees of freedom.

\section{Conclusion}

Intrinsically attached to the design of the MHM method, the a posteriori error estimator proposed in this work accounts for the multi-level approximations of the method. We restricted the numerical analysis to the first and second level MHM methods for the Stokes and Brinkman equations, for which we proved that the estimator is locally efficient and reliable in the natural norms. The results are, up to our knowledge, the first to address the impact of two-levels of approximation in a multiscale finite element method for the Stokes operator. As a result, the multiscale a posteriori error estimator yielded novel adaptivity strategies which drive first-level meshes as well as (independent) elementwise second level meshes. Fluid flow problems in highly heterogeneous validated the underlying multi-level adaptative algorithms. We showed that the MHM method on such first- and second-level adapted meshes preserves the accuracy of the velocity and pressure fields on coarse global meshes. The missing scales are upscaled through base functions computed in parallel.

\section{Appendix A. Some technical lemmas}

This section presents some technical results needed in the proof of Theorems 4.3 and 4.4.

Lemma A.1. Let $\boldsymbol{\lambda} \in \boldsymbol{\Lambda}$ and $\boldsymbol{\lambda}_{H, h} \in \boldsymbol{\Lambda}_{H}$ be the solutions of problems (3.5) and (3.13), respectively. Then

$$
\int_{K} T^{p}\left(\boldsymbol{\lambda}-\boldsymbol{\lambda}_{H, h}\right) d \boldsymbol{x}=0
$$

in the Stokes case $(\boldsymbol{\gamma}=\mathbf{0})$, and

$$
\left|\int_{K} T^{p}\left(\boldsymbol{\lambda}-\boldsymbol{\lambda}_{H, h}\right) d \boldsymbol{x}\right| \preceq H_{K}|K|^{1 / 2} \eta_{2, K}
$$

otherwise. 
Proof. Let $\boldsymbol{w}:=\frac{1}{d}\left(\boldsymbol{x}-\frac{1}{|K|} \int_{K} \boldsymbol{x} \mathrm{d} x\right)$. Noticing that $\boldsymbol{w} \in\left[H^{1}(K) \cap L_{0}^{2}(K)\right]^{d}, \nabla \cdot \boldsymbol{w}=1$, and $\nabla \boldsymbol{w}=\frac{1}{d} \mathbf{I}$, and testing (3.2) and (3.17) with $(\boldsymbol{w}, 0)$, we get

$$
\begin{aligned}
\int_{K} T^{p} \boldsymbol{\lambda}_{H, h} d \boldsymbol{x}= & \left(T^{p} \boldsymbol{\lambda}_{H, h}, \nabla \cdot \boldsymbol{w}\right)_{K}=\left(\nu \nabla T^{\boldsymbol{u}} \boldsymbol{\lambda}_{H, h}, \nabla \boldsymbol{w}\right)_{K}+\left(\boldsymbol{\gamma} T^{\boldsymbol{u}} \boldsymbol{\lambda}_{H, h}, \boldsymbol{w}\right)_{K}+\left\langle\boldsymbol{\lambda}_{H, h}, \boldsymbol{w}\right\rangle_{\partial K} \\
= & \frac{\nu}{d} \int_{K} \nabla \cdot T^{\boldsymbol{u}} \boldsymbol{\lambda}_{H, h} d \boldsymbol{x}+\left(\boldsymbol{\gamma} T^{\boldsymbol{u}} \boldsymbol{\lambda}_{H, h}, \boldsymbol{w}\right)_{K}+\left\langle\boldsymbol{\lambda}_{H, h}, \boldsymbol{w}\right\rangle_{\partial K} \\
= & \left(\gamma T^{\boldsymbol{u}} \boldsymbol{\lambda}_{H, h}, \boldsymbol{w}\right)_{K}+\left\langle\boldsymbol{\lambda}_{H, h}, \boldsymbol{w}\right\rangle_{\partial K} \\
= & \frac{\nu}{d} \int_{K} \nabla \cdot T_{h}^{\boldsymbol{u}} \boldsymbol{\lambda}_{H, h} d \boldsymbol{x}+\left(\boldsymbol{\gamma} T^{\boldsymbol{u}} \boldsymbol{\lambda}_{H, h}, \boldsymbol{w}\right)_{K}+\left\langle\boldsymbol{\lambda}_{H, h}, \boldsymbol{w}\right\rangle_{\partial K} \\
= & \left(\nu \nabla T_{h}^{u} \boldsymbol{\lambda}_{H, h}, \nabla \boldsymbol{w}\right)_{K}+\left(\boldsymbol{\gamma} T^{\boldsymbol{u}} \boldsymbol{\lambda}_{H, h}, \boldsymbol{w}\right)_{K}+\left\langle\boldsymbol{\lambda}_{H, h}, \boldsymbol{w}\right\rangle_{\partial K} \\
= & \left(T_{h}^{p} \boldsymbol{\lambda}_{H, h}, \nabla \cdot \boldsymbol{w}\right)_{K}+\left(\boldsymbol{\gamma}\left(T^{\boldsymbol{u}}-T_{h}^{\boldsymbol{u}}\right) \boldsymbol{\lambda}_{H, h}, \boldsymbol{w}\right)_{K} \\
& +\sum_{\tau \in \mathcal{T}_{h}^{K}} \kappa_{\tau}\left(-\nu \Delta T_{h}^{u} \boldsymbol{\lambda}_{H, h}+\boldsymbol{\gamma} T_{h}^{\boldsymbol{u}} \boldsymbol{\lambda}_{H, h}+\nabla T_{h}^{p} \boldsymbol{\lambda}_{H, h}, \boldsymbol{\gamma} \boldsymbol{w}\right)_{\tau} \\
= & \int_{K} T_{h}^{p} \boldsymbol{\lambda}_{H, h} d \boldsymbol{x}+\left(\boldsymbol{\gamma}\left(T^{\boldsymbol{u}}-T_{h}^{\boldsymbol{u}}\right) \boldsymbol{\lambda}_{H, h}, \boldsymbol{w}\right)_{K} \\
& +\sum_{\tau \in \mathcal{T}_{h}^{K}} \kappa_{\tau}\left(-\nu \Delta T_{h}^{u} \boldsymbol{\lambda}_{H, h}+\boldsymbol{\gamma} T_{h}^{\boldsymbol{u}} \boldsymbol{\lambda}_{H, h}+\nabla T_{h}^{p} \boldsymbol{\lambda}_{H, h}, \boldsymbol{\gamma} \boldsymbol{w}\right)_{\tau} .
\end{aligned}
$$

Proceeding in a similar way but now with the equations (3.3) and (3.18), we arrive that

$$
\begin{aligned}
\int_{K} \hat{T}^{p} \boldsymbol{f} d \boldsymbol{x}= & \left(\hat{T}^{p} \boldsymbol{f}, \nabla \cdot \boldsymbol{w}\right)_{K}=\left(\nu \nabla \hat{T}^{u} \boldsymbol{f}, \nabla \boldsymbol{w}\right)_{K}+\left(\boldsymbol{\gamma} \hat{T}^{\boldsymbol{u}} \boldsymbol{f}, \boldsymbol{w}\right)_{K}-(\boldsymbol{f}, \boldsymbol{w})_{K} \\
= & \frac{\nu}{d} \int_{K} \nabla \cdot \hat{T}^{u} \boldsymbol{f} d \boldsymbol{x}+\left(\boldsymbol{\gamma} \hat{T}^{u} \boldsymbol{f}, \boldsymbol{w}\right)_{K}-(\boldsymbol{f}, \boldsymbol{w})_{K}=\left(\boldsymbol{\gamma} \hat{T}^{u} \boldsymbol{f}, \boldsymbol{w}\right)_{K}-(\boldsymbol{f}, \boldsymbol{w})_{K} \\
= & \frac{\nu}{d} \int_{K} \nabla \cdot \hat{T}_{h}^{u} \boldsymbol{f} d \boldsymbol{x}+\left(\boldsymbol{\gamma} \hat{T}^{\boldsymbol{u}} \boldsymbol{f}, \boldsymbol{w}\right)_{K}-(\boldsymbol{f}, \boldsymbol{w})_{K} \\
= & \left(\nu \nabla \hat{T}_{h}^{u} \boldsymbol{f}, \nabla \boldsymbol{w}\right)_{K}+\left(\boldsymbol{\gamma} \hat{T}^{\boldsymbol{u}} \boldsymbol{f}, \boldsymbol{w}\right)_{K}-(\boldsymbol{f}, \boldsymbol{w})_{K} \\
= & \left(\hat{T}_{h}^{p} \boldsymbol{f}, \nabla \cdot \boldsymbol{w}\right)_{K}+\left(\boldsymbol{\gamma}\left(\hat{T}^{\boldsymbol{u}}-\hat{T}_{h}^{\boldsymbol{u}}\right) \boldsymbol{f}, \boldsymbol{w}\right)_{K} \\
& +\sum_{\tau \in \mathcal{T}_{h}^{K}} \kappa_{\tau}\left(-\nu \Delta \hat{T}_{h}^{u} \boldsymbol{f}+\boldsymbol{\gamma} \hat{T}_{h}^{u} \boldsymbol{f}+\nabla \hat{T}_{h}^{p} \boldsymbol{f}-\boldsymbol{f}, \boldsymbol{\gamma} \boldsymbol{w}\right)_{\tau} \\
= & \int_{K} \hat{T}_{h}^{p} \boldsymbol{f} d \boldsymbol{x}+\left(\gamma\left(\hat{T}^{\boldsymbol{u}}-\hat{T}_{h}^{\boldsymbol{u}}\right) \boldsymbol{f}, \boldsymbol{w}\right)_{K} \\
& +\sum_{\tau \in \mathcal{T}_{h}^{K}} \kappa_{\tau}\left(-\nu \Delta \hat{T}_{h}^{u} \boldsymbol{f}+\boldsymbol{\gamma} \hat{T}_{h}^{u} \boldsymbol{f}+\nabla \hat{T}_{h}^{p} \boldsymbol{f}-\boldsymbol{f}, \boldsymbol{\gamma} \boldsymbol{w}\right)_{\tau}
\end{aligned}
$$


and then, from (A.1), (A.2) and the third equations in (3.5) and (3.13), it holds

$$
\begin{aligned}
\int_{K} T^{p}\left(\boldsymbol{\lambda}-\boldsymbol{\lambda}_{H, h}\right) d \boldsymbol{x}= & \int_{K} T^{p} \boldsymbol{\lambda} d \boldsymbol{x}-\int_{K} T^{p} \boldsymbol{\lambda}_{H, h} d \boldsymbol{x}=-\int_{K} \hat{T}^{p} \boldsymbol{f} d \boldsymbol{x}-\int_{K} T^{p} \boldsymbol{\lambda}_{H, h} d \boldsymbol{x} \\
= & -\int_{K} \hat{T}^{p} \boldsymbol{f} d \boldsymbol{x}-\left[\int_{K} T_{h}^{p} \boldsymbol{\lambda}_{H, h} d \boldsymbol{x}+\left(\boldsymbol{\gamma}\left(T^{\boldsymbol{u}}-T_{h}^{\boldsymbol{u}}\right) \boldsymbol{\lambda}_{H, h}, \boldsymbol{w}\right)_{K}\right. \\
& \left.+\sum_{\tau \in \mathcal{T}_{h}^{K}} \kappa_{\tau}\left(-\nu \Delta T_{h}^{\boldsymbol{u}} \boldsymbol{\lambda}_{H, h}+\boldsymbol{\gamma} T_{h}^{\boldsymbol{u}} \boldsymbol{\lambda}_{H, h}+\nabla T_{h}^{p} \boldsymbol{\lambda}_{H, h}, \boldsymbol{\gamma} \boldsymbol{w}\right)_{\tau}\right] \\
= & -\int_{K} \hat{T}_{h}^{p} \boldsymbol{f} d \boldsymbol{x}-\left(\boldsymbol{\gamma}\left(\hat{T}^{\boldsymbol{u}}-\hat{T}_{h}^{\boldsymbol{u}}\right) \boldsymbol{f}, \boldsymbol{w}\right)_{K} \\
& -\sum_{\tau \in \mathcal{T}_{h}^{K}} \kappa_{\tau}\left(-\nu \Delta \hat{T}_{h}^{\boldsymbol{u}} \boldsymbol{f}+\gamma \hat{T}_{h}^{\boldsymbol{u}} \boldsymbol{f}+\nabla \hat{T}_{h}^{p} \boldsymbol{f}-\boldsymbol{f}, \boldsymbol{\gamma} \boldsymbol{w}\right)_{\tau} \\
& -\left[\int_{K} T_{h}^{p} \boldsymbol{\lambda}_{H, h} d \boldsymbol{x}+\left(\boldsymbol{\gamma}\left(T^{\boldsymbol{u}}-T_{h}^{\boldsymbol{u}}\right) \boldsymbol{\lambda}_{H, h}, \boldsymbol{w}\right)_{K}\right. \\
& \left.+\sum_{\tau \in \mathcal{T}_{h}^{K}} \kappa_{\tau}\left(-\nu \Delta T_{h}^{\boldsymbol{u}} \boldsymbol{\lambda}_{H, h}+\boldsymbol{\gamma} T_{h}^{\boldsymbol{u}} \boldsymbol{\lambda}_{H, h}+\nabla T_{h}^{p} \boldsymbol{\lambda}_{H, h}, \boldsymbol{\gamma} \boldsymbol{w}\right)_{\tau}\right] \\
= & -\left(\boldsymbol{\gamma}\left(T^{\boldsymbol{u}}-T_{h}^{\boldsymbol{u}}\right) \boldsymbol{\lambda}_{H, h}, \boldsymbol{w}\right)_{K}-\left(\boldsymbol{\gamma}\left(\hat{T}^{\boldsymbol{u}}-\hat{T}_{h}^{\boldsymbol{u}}\right) \boldsymbol{f}, \boldsymbol{w}\right)_{K} \\
& +\sum_{\tau \in \mathcal{T}_{h}^{K}} \kappa_{\tau}\left(-\nu \Delta \boldsymbol{u}_{H, h}+\gamma \boldsymbol{u}_{H, h}+\nabla p_{H, h}-\boldsymbol{f}, \boldsymbol{\gamma}\right)_{\tau} \\
= & -\left(\boldsymbol{\gamma}\left(T^{\boldsymbol{u}}-T_{h}^{\boldsymbol{u}}\right) \boldsymbol{\lambda}_{H, h}, \boldsymbol{w}\right)_{K}-\left(\boldsymbol{\gamma}\left(\hat{T}^{\boldsymbol{u}}-\hat{T}_{h}^{\boldsymbol{u}}\right) \boldsymbol{f}, \boldsymbol{w}\right)_{K}+\sum_{\tau \in \mathcal{T}_{h}^{K}} \kappa_{\tau}\left(\boldsymbol{R}_{\tau}^{K}, \boldsymbol{\gamma} \boldsymbol{w}\right)_{\tau} .
\end{aligned}
$$

Observe that for the Stokes problem $(\boldsymbol{\gamma}=\mathbf{0})$, equation (A.3) vanishes and the result follows. For the Brinkman case, applying Cauchy-Schwartz's inequality, Lemma 4.1, using (4.11) and recalling from [11, Theorem 3.2] that, since $\boldsymbol{w} \in\left[H^{1}(K) \cap L_{0}^{2}(K)\right]^{d}$ and the convexity of $K$, we have that

the result follows.

$$
\|\boldsymbol{w}\|_{0, K} \leq \frac{H_{K}}{\pi}|\boldsymbol{w}|_{1, K}=\frac{1}{d \pi} H_{K}|K|^{1 / 2}
$$

The second auxiliary result establishes bounds for the local pressure associated to the error $\boldsymbol{\lambda}-\boldsymbol{\lambda}_{H, h}$ in terms of its velocity counterpart.

Lemma A.2. Let $\boldsymbol{\lambda} \in \boldsymbol{\Lambda}$ and $\boldsymbol{\lambda}_{H, h} \in \boldsymbol{\Lambda}_{H}$ be the solutions of problems (3.5) and (3.13), respectively. Then, we have that

for the Stokes problem, and

$$
\left\|T^{p}\left(\boldsymbol{\lambda}-\boldsymbol{\lambda}_{H, h}\right)\right\|_{Q(K)} \preceq\left\|T^{\boldsymbol{u}}\left(\boldsymbol{\lambda}-\boldsymbol{\lambda}_{H, h}\right)\right\|_{\mathbf{V}(K)}
$$

$$
\left\|T^{p}\left(\boldsymbol{\lambda}-\boldsymbol{\lambda}_{H, h}\right)\right\|_{Q(K)} \preceq\left\|T^{\boldsymbol{u}}\left(\boldsymbol{\lambda}-\boldsymbol{\lambda}_{H, h}\right)\right\|_{\mathbf{v}(K)}+H_{K} \eta_{2, K}
$$

for the Brinkman problem.

Proof. Let $\boldsymbol{\mu}:=\boldsymbol{\lambda}-\boldsymbol{\lambda}_{H, h}$ and consider the Stokes case. From Lemma A.1, the function $T^{p} \boldsymbol{\mu} \in L_{0}^{2}(K)$ and then there exists $\tilde{\boldsymbol{w}} \in H_{0}^{1}(K)^{d}$ such that

$$
\nabla \cdot \tilde{\boldsymbol{w}}=T^{p} \boldsymbol{\mu} \text { in } K
$$

satisfying

$$
|\tilde{\boldsymbol{w}}|_{1, K} \preceq\left\|T^{p} \boldsymbol{\mu}\right\|_{0, K}
$$

Next, defined $\boldsymbol{w}:=\tilde{\boldsymbol{w}}-\frac{1}{|K|} \tilde{\boldsymbol{w}} \in \mathbf{V}_{0}^{\perp}(K)$. From the first equation of (3.5) and (3.13), and the local problems (3.2) and (3.3), it holds

$$
\begin{aligned}
0 & =-\langle\boldsymbol{\mu}, \tilde{\boldsymbol{w}}\rangle_{\partial K}=-\langle\boldsymbol{\mu}, \boldsymbol{w}\rangle_{\partial K} \\
& =a_{K}\left(T^{\boldsymbol{u}} \boldsymbol{\mu}, \boldsymbol{w}\right)+b_{K}\left(\boldsymbol{w}, T^{p} \boldsymbol{\mu}\right)=a_{K}\left(T^{\boldsymbol{u}} \boldsymbol{\mu}, \tilde{\boldsymbol{w}}\right)+b_{K}\left(\tilde{\boldsymbol{w}}, T^{p} \boldsymbol{\mu}\right) .
\end{aligned}
$$


Hence, from (A.4), (A.5), (A.6), and Cauchy-Schwartz's inequality, we get

$$
\left\|T^{p} \boldsymbol{\mu}\right\|_{Q(K)}^{2}=\left(T^{p} \boldsymbol{\mu}, \nabla \cdot \tilde{\boldsymbol{w}}\right)_{K}=-b_{K}\left(\tilde{\boldsymbol{w}}, T^{p} \boldsymbol{\mu}\right)=a_{K}\left(T^{\boldsymbol{u}} \boldsymbol{\mu}, \tilde{\boldsymbol{w}}\right) \preceq\left\|T^{p} \boldsymbol{\mu}\right\|_{Q(K)}\left\|T^{\boldsymbol{u}} \boldsymbol{\mu}\right\|_{\mathbf{V}(K)}
$$

and the result follows.

Now, we proceed to the Brinkman case following the proof for the Stokes one. First, we notice that $T^{p} \boldsymbol{\mu}$ is not necessarily an element of $L_{0}^{2}(K)$. Then, we decompose

$$
T^{p} \boldsymbol{\mu}=\tilde{q}+q_{0} \quad \text { where } \quad \tilde{q} \in L_{0}^{2}(K) \text { and } q_{0}=\frac{1}{|K|} \int_{K} T^{p} \boldsymbol{\mu} \mathrm{d} x,
$$

and pick $\tilde{\boldsymbol{w}} \in H_{0}^{1}(K)^{d}$ such that

$$
\nabla \cdot \tilde{\boldsymbol{w}}=\tilde{q} \quad \text { in } K
$$

and

$$
|\tilde{\boldsymbol{w}}|_{1, K} \preceq\|\tilde{q}\|_{0, K}
$$

Also, from (A.7) it holds

$$
\|\tilde{q}\|_{Q(K)} \leq\left\|T^{p} \boldsymbol{\mu}\right\|_{Q(K)} .
$$

From local problems (3.2) and (3.3), we arrive at

$$
0=-\langle\boldsymbol{\mu}, \tilde{\boldsymbol{w}}\rangle_{\partial K}=a_{K}\left(T^{\boldsymbol{u}} \boldsymbol{\mu}, \tilde{\boldsymbol{w}}\right)+b_{K}\left(\tilde{\boldsymbol{w}}, T^{p} \boldsymbol{\mu}\right)
$$

and then, from (A.8), (A.9), (A.11), Cauchy-Schwartz's inequality, (A.10) and Lemma A.1, we get

$$
\begin{aligned}
\left\|T^{p} \boldsymbol{\mu}\right\|_{Q(K)}^{2} & =\left(T^{p} \boldsymbol{\mu}, \tilde{q}\right)_{K}+\left(T^{p} \boldsymbol{\mu}, q_{0}\right)_{K}=\left(T^{p} \boldsymbol{\mu}, \nabla \cdot \tilde{\boldsymbol{w}}\right)_{K}+\left(T^{p} \boldsymbol{\mu}, q_{0}\right)_{K} \\
& =-b_{K}\left(\tilde{\boldsymbol{w}}, T^{p} \boldsymbol{\mu}\right)+\left(T^{p} \boldsymbol{\mu}, q_{0}\right)_{K}=a_{K}\left(T^{\boldsymbol{u}} \boldsymbol{\mu}, \tilde{\boldsymbol{w}}\right)+\left(T^{p} \boldsymbol{\mu}, q_{0}\right)_{K} \\
& \preceq\left\|T^{\boldsymbol{u}} \boldsymbol{\mu}\right\|_{\mathbf{V}(K)}\|\tilde{q}\|_{Q(K)}+\left\|T^{p} \boldsymbol{\mu}\right\|_{Q(K)}\left\|q_{0}\right\|_{Q(K)} \\
& \preceq\left\|T^{\boldsymbol{u}} \boldsymbol{\mu}\right\|_{\mathbf{V}(K)}\left\|T^{p} \boldsymbol{\mu}\right\|_{Q(K)}+\left\|T^{p} \boldsymbol{\mu}\right\|_{Q(K)}\left\|q_{0}\right\|_{Q(K)} \\
& =\left(\left\|T^{\boldsymbol{u}} \boldsymbol{\mu}\right\|_{\mathbf{V}(K)}+\left\|q_{0}\right\|_{0, K}\right)\left\|T^{p} \boldsymbol{\mu}\right\|_{Q(K)} \preceq\left(\left\|T^{\boldsymbol{u}} \boldsymbol{\mu}\right\|_{\mathbf{V}(K)}+H_{K} \eta_{2, K}\right)\left\|T^{p} \boldsymbol{\mu}\right\|_{Q(K)}
\end{aligned}
$$

and the result follows.

Now, we detail an intermediate error estimate for the local velocity associated to $\boldsymbol{\lambda}-\boldsymbol{\lambda}_{H, h}$ with respect to $\eta_{1}$ and $\eta_{2}$.

Lemma A.3. Let $\boldsymbol{\lambda} \in \boldsymbol{\Lambda}$ and $\boldsymbol{\lambda}_{H, h} \in \boldsymbol{\Lambda}_{H}$ be the solutions of problems (3.5) and (3.13), respectively. The following estimates hold

$$
\left\|T^{\boldsymbol{u}}\left(\boldsymbol{\lambda}-\boldsymbol{\lambda}_{H, h}\right)\right\|_{\mathbf{v}} \preceq \begin{cases}\eta_{1}+\eta_{2} & \text { if } \boldsymbol{\gamma}=\mathbf{0}, \\ \eta_{1}+\eta_{2}+H\left\{\left(\eta_{1}+\eta_{2}\right) \eta_{2}\right\}^{1 / 2} & \text { otherwise. }\end{cases}
$$

Proof. Let $\boldsymbol{\mu}=\boldsymbol{\lambda}-\boldsymbol{\lambda}_{H, h}$. From (2.2), using $\left(T^{p} \boldsymbol{\mu}, \nabla \cdot T^{\boldsymbol{u}} \boldsymbol{\mu}\right)_{\mathcal{T}_{H}}=0$ and (3.2), we obtain that

$$
-\left(\boldsymbol{\mu}, T^{\boldsymbol{u}} \boldsymbol{\mu}\right)_{\partial \mathcal{T}_{H}}=\sum_{K \in \mathcal{T}_{H}} B_{K}\left(\left(T^{\boldsymbol{u}} \boldsymbol{\mu}, T^{p} \boldsymbol{\mu}\right),\left(T^{\boldsymbol{u}} \boldsymbol{\mu}, 0\right)\right) \geq C\left\|T^{\boldsymbol{u}} \boldsymbol{\mu}\right\|_{\mathbf{V}}^{2},
$$

where $C$ depends only on $\nu, \gamma_{\max }$ and $d_{\Omega}$. Using $\left(\boldsymbol{\mu}, \boldsymbol{v}_{0}\right)_{\partial \mathcal{T}_{H}}=0$, for all $\boldsymbol{v}_{0} \in \mathbf{V}_{0}$, and from (2.2), (2.12), (3.2) and the orthogonality between functions in $\mathbf{V}$ and $\mathbf{V}_{0}^{\perp}$, it holds

$$
\begin{aligned}
\frac{\sqrt{2}}{2}\|\boldsymbol{\mu}\|_{\boldsymbol{\Lambda}} & \leq \sup _{\boldsymbol{v} \in \mathbf{V}} \frac{(\boldsymbol{\mu}, \boldsymbol{v})_{\partial \mathcal{T}_{H}}}{\|\boldsymbol{v}\|_{\mathbf{V}}}=\sup _{v_{0} \in \mathbf{V}_{0}, \boldsymbol{v}_{0}^{\perp} \in \mathbf{V}_{0}^{\perp}} \frac{\left(\boldsymbol{\mu}, \boldsymbol{v}_{0}^{\perp}\right) \partial \mathcal{T}_{H}}{\left\|\boldsymbol{v}_{0}+\boldsymbol{v}_{0}^{\perp}\right\|_{\mathbf{V}}} \\
& \leq \sup _{\boldsymbol{v}_{0}^{\perp} \in \mathbf{V}_{0}^{\perp}} \frac{\left(\boldsymbol{\mu}, \boldsymbol{v}_{0}^{\perp}\right)_{\partial \mathcal{T}_{H}}}{\left\|\boldsymbol{v}_{0}^{\perp}\right\|_{\mathbf{V}}}=\sup _{\boldsymbol{v}_{0}^{\perp} \in \mathbf{V}_{0}^{\perp}} \frac{-B\left(\left(T^{\boldsymbol{u}} \boldsymbol{\mu}, T^{p} \boldsymbol{\mu}\right),\left(\boldsymbol{v}_{0}^{\perp}, 0\right)\right.}{\left\|\boldsymbol{v}_{0}^{\perp}\right\|_{\mathbf{V}}} \\
& \preceq\left\|T^{\boldsymbol{u}} \boldsymbol{\mu}\right\|_{\mathbf{V}}+\left\|T^{p} \boldsymbol{\mu}\right\|_{Q} .
\end{aligned}
$$

Thus, from Lemma A.2 we get

$$
\|\boldsymbol{\mu}\|_{\boldsymbol{\Lambda}} \preceq \begin{cases}\left\|T^{\boldsymbol{u}} \boldsymbol{\mu}\right\|_{\mathbf{V}} & \text { if } \boldsymbol{\gamma}=\mathbf{0}, \\ \left\|T^{\boldsymbol{u}} \boldsymbol{\mu}\right\|_{\mathbf{V}}+H \eta_{2} & \text { otherwise. }\end{cases}
$$


Now, using again $\left(\boldsymbol{\mu}, \boldsymbol{u}_{0}\right)_{\partial \mathcal{T}_{H}}=\left(\boldsymbol{\mu}, \boldsymbol{u}_{0}^{H, h}\right)_{\partial \mathcal{T}_{H}}=0$, from (3.6), (3.20) and Lemmas 4.1 and 4.2, we obtain

$$
\begin{aligned}
-\left(\boldsymbol{\mu}, T^{\boldsymbol{u}} \boldsymbol{\mu}\right)_{\partial \mathcal{T}_{H}} & =-\left(\boldsymbol{\mu}, T^{\boldsymbol{u}}\left(\boldsymbol{\lambda}-\boldsymbol{\lambda}_{H, h}\right)\right)_{\partial \mathcal{T}_{H}}=-\left(\boldsymbol{\mu}, \boldsymbol{u}_{0}+T^{\boldsymbol{u}} \boldsymbol{\lambda}+\hat{T}^{\boldsymbol{u}} \boldsymbol{f}-\left(T^{\boldsymbol{u}} \boldsymbol{\lambda}_{H, h}+\hat{T}^{u} \boldsymbol{f}\right)\right)_{\partial \mathcal{T}_{H}} \\
& =-(\boldsymbol{\mu}, \boldsymbol{u})_{\partial \mathcal{T}_{H}}+\left(\boldsymbol{\mu}, T^{\boldsymbol{u}} \boldsymbol{\lambda}_{H, h}+\hat{T}^{\boldsymbol{u}} \boldsymbol{f}\right)_{\partial \mathcal{T}_{H}} \\
& =-\langle\boldsymbol{\mu}, \boldsymbol{g}\rangle_{\partial \Omega}+\left(\boldsymbol{\mu}, \boldsymbol{u}_{0}^{H, h}+T_{h}^{\boldsymbol{u}} \boldsymbol{\lambda}_{H, h}+\hat{T}_{h}^{\boldsymbol{u}} \boldsymbol{f}+\left(T^{\boldsymbol{u}}-T_{h}^{\boldsymbol{u}}\right) \boldsymbol{\lambda}_{H, h}+\left(\hat{T}^{\boldsymbol{u}}-\hat{T}_{h}^{\boldsymbol{u}}\right) \boldsymbol{f}\right)_{\partial \mathcal{T}_{H}} \\
& =-\langle\boldsymbol{\mu}, \boldsymbol{g}\rangle_{\partial \Omega}+\left(\boldsymbol{\mu}, \boldsymbol{u}_{H, h}\right)_{\partial \mathcal{T}_{H}}+\left(\boldsymbol{\mu},\left(T^{\boldsymbol{u}}-T_{h}^{\boldsymbol{u}}\right) \boldsymbol{\lambda}_{H, h}+\left(\hat{T}^{\boldsymbol{u}}-\hat{T}_{h}^{\boldsymbol{u}}\right) \boldsymbol{f}\right)_{\partial \mathcal{T}_{H}} \\
& =-(\boldsymbol{\mu}, \boldsymbol{\chi})_{\partial \mathcal{T}_{H}}+\left(\boldsymbol{\mu},\left(T^{\boldsymbol{u}}-T_{h}^{\boldsymbol{u}}\right) \boldsymbol{\lambda}_{H, h}+\left(\hat{T}^{\boldsymbol{u}}-\hat{T}_{h}^{\boldsymbol{u}}\right) \boldsymbol{f}\right)_{\partial \mathcal{T}_{H}} \\
& \leq\left(\|\boldsymbol{\chi}\|_{\mathbf{V}}+\left\|\left(T^{\boldsymbol{u}}-T_{h}^{\boldsymbol{u}}\right) \boldsymbol{\lambda}_{H, h}+\left(\hat{T}^{\boldsymbol{u}}-\hat{T}_{h}^{\boldsymbol{u}}\right) \boldsymbol{f}\right\|_{\mathbf{V}}\right)\|\boldsymbol{\mu}\|_{\boldsymbol{\Lambda}} \\
& \preceq\left(\eta_{1}+\left\|\left(T^{\boldsymbol{u}}-T_{h}^{\boldsymbol{u}}\right) \boldsymbol{\lambda}_{H, h}+\left(\hat{T}^{\boldsymbol{u}}-\hat{T}_{h}^{\boldsymbol{u}}\right) \boldsymbol{f}\right\|_{\mathbf{V}}\right)\|\boldsymbol{\mu}\|_{\boldsymbol{\Lambda}} \\
& \preceq\left(\eta_{1}+\eta_{2}\right)\|\boldsymbol{\mu}\|_{\boldsymbol{\Lambda}} .
\end{aligned}
$$

Hence, for the Stokes equations $(\boldsymbol{\gamma}=\mathbf{0})$ we use (A.12), (A.13) and (A.14) to get the desired result. For the Brinkman equation, we use again (A.12), (A.13) and (A.14), to obtain that

$$
\begin{aligned}
\left\|T^{\boldsymbol{u}} \boldsymbol{\mu}\right\|_{\mathbf{V}}^{2} & \preceq\left(\eta_{1}+\eta_{2}\right)\left(\left\|T^{\boldsymbol{u}} \boldsymbol{\mu}\right\|_{\mathbf{V}}+H^{2} \eta_{2}\right) \\
& \preceq\left(\eta_{1}+\eta_{2}\right)^{2}+\frac{1}{2}\left\|T^{\boldsymbol{u}} \boldsymbol{\mu}\right\|_{\mathbf{V}}^{2}+\left(\eta_{1}+\eta_{2}\right) H^{2} \eta_{2},
\end{aligned}
$$

thus

and the result follows.

$$
\left\|T^{\boldsymbol{u}} \boldsymbol{\mu}\right\|_{\mathbf{v}} \preceq\left(\eta_{1}+\eta_{2}\right)+H\left\{\left(\eta_{1}+\eta_{2}\right) \eta_{2}\right\}^{1 / 2}
$$

\section{REFERENCES}

[1] A. Abdulle and A. Nonnenmacher. A posteriori error estimates in quantities of interest for the finite element heterogeneous multiscale method. Numer. Methods for Partial Differential Equations, 29(5):1629-1656, 2013.

[2] S. Agmon. Lectures on Elliptic Boundary Value Problems, volume 369. AMS Chelsea Publishing, Providence, 2010.

[3] A. Agouzal and J. M. Thomas. Une méthode d'eléments finis hybrides en décomposition de domaines. RAIRO-Math Model Num., 29(6):749-765, 1995.

[4] M. Ainsworth and J. T. Oden. A posteriori error estimation in finite element analysis. Pure and Applied Mathematics. Wiley-Interscience, New York, 2000.

[5] R. Araya, G. R. Barrenechea, and F. Valentin. A stabilized finite-element method for the Stokes problem including element and edge residuals. IMA J. Numer. Anal., 27(1):172-197, 2007.

[6] R. Araya, C. Harder, D. Paredes, and F. Valentin. Multiscale hybrid-mixed method. SIAM J. Numer. Anal., 51(6):35053531,2013

[7] R. Araya, C. Harder, A. Poza, and F. Valentin. The multiscale hybrid-mixed method for the Stokes and Brinkman equations - The method. Comput. Methods Appl. Mech. Engrg., 324:29-53, 2017.

[8] I. Babuska and E. Osborn. Generalized finite element methods: Their performance and their relation to mixed methods. SIAM J. Num. Anal., 20(3):510-536, 1983.

[9] J. Baiges and R. Codina. Variational multiscale error estimators for solid mechanics adaptive simulations: An orthogonal subgrid scale approach. Comput. Methods Appl. Mech. Engrg., 325:37 - 55, 2017.

[10] G. R. Barrenechea and F. Valentin. An unusual stabilized finite element method for a generalized Stokes problem. Numer. Math., 92(4):653-677, 2002.

[11] M Bebendorf. A note on the Poincaré inequality for convex domains. Z. Anal. Anwend, 22(4):751-756, 2003.

[12] F. Brezzi and D. Marini. Error estimates for the three-field formulation with bubble stabilization. Math. Comp., 70(235):911-934, 2000.

[13] F. Brezzi and L. D. Marini. A three-field domain decomposition method. In Domain decomposition methods in science and engineering (Como, 1992), volume 157 of Contemp. Math., pages 27-34. Amer. Math. Soc., Providence, RI, Providence, 1994.

[14] L. Chamoin and F. Legoll. A posteriori error estimation and adaptive strategy for the control of MsFEM computations. Comput. Methods Appl. Mech. Engrg., 336:1-38, 2018.

[15] M. A. Christie and M. J. Blunt. Tenth SPE comparative solution project: A comparison of upscaling techniques. $P E$ Reservoir Eval. Eng., pages 308-317, 2001.

[16] Ph. Clément. Approximation by finite element functions using local regularization. R.A.I.R.O. Anal. Numer., 9:77-84, 1975.

[17] R. G. Durán. An elementary proof of the continuity from $L_{0}^{2}(\Omega)$ to $H_{0}^{1}(\Omega)^{n}$ of Bogovskii's right inverse of the divergence. Rev. Un. Mat. Argentina, 53(2):59-78, 2012.

[18] Y. Efendiev, J. Galvis, and T.Y. Hou. Generalized multiscale finite element methods (GMsFEM). J. Comput. Phys., 251:116-135, 2013. 
[19] A. Ern and J.-L. Guermond. Theory and practice of finite elements, volume 159 of Applied Mathematical Sciences. SpringerVerlag, New York, 2004.

[20] L. Franca and A Madureira. Element diameter free stability parameters for stabilized methods applied to fluids. Comput. Methods Appl. Mech. Engrg., 105(3):395-403, 1993.

[21] V. Girault and P.-A. Raviart. Finite element methods for Navier-Stokes equations, volume 5 of Springer Series in Computational Mathematics. Springer-Verlag, Berlin, 1986. Theory and algorithms.

[22] P. Grisvard. Singularities in boundary value problems. Masson, Paris, 1992.

[23] I. Harari and T. Hughes. What are $\mathrm{C}$ and h?: Inequalities for the analysis and design of finite element methods. Comput. Methods Appl. Mech. Engrg., 97(2):157-192, 1992.

[24] C. Harder, A .L. Madureira, and F. Valentin. A hybrid-mixed method for elasticity. ESAIM: Math. Model. Num. Anal., 50(2):311-336, 2016.

[25] C. Harder, D. Paredes, and F. Valentin. A family of multiscale hybrid-mixed finite element methods for the Darcy equation with rough coefficients. J. Comput. Phys., 245:107-130, 2013.

[26] C. Harder, D. Paredes, and F. Valentin. On a multiscale hybrid-mixed method for advective-reactive dominated problems with heterogenous coefficients. SIAM Multiscale Model. and Simul., 13(2):491-518, 2015.

[27] P. Henning and M. Ohlberger. A-posteriori error estimate for a heterogeneous multiscale approximation of advectiondiffusion problems with large expected drift. Discrete Contin. Dyn. Syst. Ser. S, 9(5):1393-1420, 2016.

[28] T. Y. Hou, X. Wu, and Z. Cai. Convergence of a multiscale finite element method for elliptic problems with rapidly oscillating coefficients. Math. Comp., 68(227):913-943, 1999

[29] T. J.R. Hughes. Multiscale phenomena: Green's functions, the Dirichlet-to-Neumann formulation, subgrid scale models, bubbles and the origins of stabilized methods. Comput. Methods Appl. Mech. Engrg., 127(1-4):387-401, 1995.

[30] D. Irisarri and G. Hauke. A posteriori pointwise error computation for 2-D transport equations based on the variational multiscale method. Comput. Methods Appl. Mech. Engrg., 311:648 - 670, 2016.

[31] S. Lanteri, D. Paredes, C. Scheid, and F. Valentin. The multiscale hybrid-mixed method for the maxwell equations in heterogeneous media. SIAM Multiscale Model. Simul., 16(4):1648-1683, 2018.

[32] A. Målqvist and D. Peterseim. Localization of elliptic multiscale problems. Math. Comp., 83(290):2583-2603, 2014.

[33] M. Ohlberger. A posteriori error estimates for the heterogeneous multiscale finite element method for elliptic homogenization problems. Multiscale Model. Simul., 4(1):88-114, 2005.

[34] G. Pencheva, M. Vohralík, M. Wheeler, and T. Wildey. Robust a posteriori error control and adaptivity for multiscale, multinumerics, and mortar coupling. SIAM J. Numer. Anal., 51(1):526-554, 2013.

[35] P.A. Raviart and J.M. Thomas. Primal hybrid finite element methods for 2nd order elliptic equations. Math. Comp., 31(138):391-413, 1977.

[36] S. Tavener and T. Wildey. Adjoint based a posteriori analysis of multiscale mortar discretizations with multinumerics. SIAM J. Sci. Comput., 35(6):A2621-A2642, 2013.

[37] Rüdiger Verfürth. A review of a posteriori error estimation and adaptive mesh-refinement techniques. John Wiley \& Sons Inc, Chichester, 1996

[38] E. Weinan and B. Engquist. The heterogeneous multiscale methods. Commun. Math. Sci., 1(1):87-132, 2003.

(R. Araya and R. Rebolledo) Departamento de Ingeniería Matemática \& CI ${ }^{2}$ MA, Universidad de Concepción, Casilla 160-C, Concepción, Chile

Email address: rodolfo.araya@udec.cl, ramirorebolledo@udec.cl

Department of Mathematical and Computational Methods, National Laboratory for Scientific Computing LnCC, Av. Getúlio Vargas, 333, 25651-070 Petrópolis - RJ, Brazil

Email address: valentin@lncc.br 